Article

\title{
Synthesis, Biological Evaluation and Docking Studies of Chalcone and Flavone Analogs as Antioxidants and Acetylcholinesterase Inhibitors
}

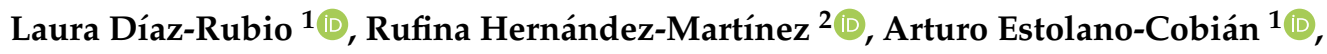 \\ Daniel Chávez-Velasco ${ }^{3}{ }^{(1)}$, Ricardo Salazar-Aranda ${ }^{4}$, Noemí Waksman de Torres ${ }^{4}$, \\ Ignacio A. Rivero ${ }^{3}$, Víctor García-González ${ }^{5} \mathbb{D}$, Marco A. Ramos ${ }^{1, * \mathbb{D}}$ and \\ Iván Córdova-Guerrero ${ }^{1, * \mathbb{D}}$ \\ 1 Facultad de Ciencias Químicas e Ingeniería, Universidad Autónoma de Baja California, \\ Tijuana, B.C. 22390, México; ldiaz26@uabc.edu.mx (L.D.-R.); arturo.estolano@uabc.edu.mx (A.E.-C.) \\ 2 Departamento de Microbiología, Centro de Investigación Científica y de Educación Superior de Ensenada, \\ Ensenada, B.C. 22860, México; ruhernan@cicese.mx \\ 3 Centro de Graduados e Investigación en Química, Tecnológico Nacional de México/Instituto Tecnológico de \\ Tijuana, Tijuana, B.C. 22510, México; dchavez@tectijuana.mx (D.C.-V.); irivero@tectijuana.mx (I.A.R.) \\ 4 Departamento de Química Analítica, Facultad de Medicina, Universidad Autónoma de Nuevo León, \\ San Nicolas de los Garza, Monterrey 64460, México; salazar121212@yahoo.com (R.S.-A.); \\ nwaksman@gmail.com (N.W.d.T.) \\ 5 Facultad de Medicina, Universidad Autónoma de Baja California, Mexicali, B.C. 21000, México; \\ vgarcia62@uabc.edu.mx \\ * Correspondence: mramos@uabc.edu.mx (M.A.R.); icordova@uabc.edu.mx (I.C.-G.); \\ Tel.: +52-664-120-7741 (I.C.-G.)
}

Received: 27 December 2018; Accepted: 21 January 2019; Published: 26 January 2019

\begin{abstract}
Several oxidative processes are related to a wide range of human chronic and degenerative diseases, like Alzheimer's disease, which also has been related to cholinergic processes. Therefore, search for new or improved antioxidant molecules with acetylcholinesterase activity is essential to offer alternative chemotherapeutic agents to support current drug therapies. A series of chalcone (2a-2k) and flavone (3a-3k) analogs were synthesized, characterized, and evaluated as acetylcholinesterase (AChE) inhibitors, and antioxidant agents using 1,1-diphenyl-2-picrylhydrazyl (DPPH•), 2-2'-azino-bis-(3-ethylbenzothiazoline-6-sulfonate) (ABTS•), and $\beta$-carotene/linoleic acid bleaching assay. Compounds more active were $3 \mathbf{j}$ and $\mathbf{2 k}$ in DPPH with $\mathrm{EC}_{50}$ of $1 \times 10^{-8}$ and $5.4 \times 10^{-3} \mu \mathrm{g} / \mathrm{mL}$, respectively; $2 \mathrm{~g}$ and $3 \mathbf{i}$ in ABTS $\left(1.14 \times 10^{-2}\right.$ and $\left.1.9 \times 10^{-3} \mu \mathrm{g} / \mathrm{mL}\right) ; 2 \mathrm{e}, 2 \mathrm{f}$, $\mathbf{3 f}, \mathbf{2} \mathbf{j}$, and $\mathbf{3} \mathbf{j}$ exceeded the $\alpha$-tocopherol control in the $\beta$-carotene assay $(98-99 \%$ of antioxidant activity). At acetylcholinesterase inhibition assay, flavones were more active than chalcones; the best results were compounds $\mathbf{2} \mathbf{d}$ and $\mathbf{3} \mathbf{d}\left(\mathrm{IC}_{50} 21.5\right.$ and $26.8 \mu \mathrm{g} / \mathrm{mL}$, respectively), suggesting that the presence of the nitro group enhances the inhibitory activity. The docking of these two structures were made to understand their interactions with the $\mathrm{AChE}$ receptor. Although further in vivo testing must be performed, our results represent an important step towards the identification of improved antioxidants and acetylcholinesterase inhibitors.
\end{abstract}

Keywords: chalcone; flavone; antioxidant activities; DPPH; acetylcholinesterase; docking

\section{Introduction}

Collectively, radical and non-radical species formed by the partial reduction of oxygen are known as reactive oxygen species (ROS) [1]. When ROS overcame the cellular antioxidant defense system, 
whether through an increase in ROS levels or a decrease in the cellular antioxidant capacity, oxidative stress occurs [2], which disturbs cellular metabolism, damages cellular constituents, and triggers the activation of specific signaling pathways [3]. The pathogenesis of several chronic and degenerative human diseases, including cardiovascular, neurodegenerative, and cancerous ones, has been linked to a cellular condition exhibiting oxidative stress $[4,5]$. To counteract this stressful condition, the human body has several mechanisms and produces a broad variety of antioxidants [6], which together quench ROS activities and reduce cellular damage [7].

Oxidative stress plays a crucial role in the etiology and pathogenesis of neurodegenerative diseases, like Alzheimer [8]. Alzheimer's disease (AD) is globally the most common cause of dementia in adults above 60 years [9]; ageing lowers the antioxidant systems activity, leading to free radical progressive accumulation, triggering lipid peroxidation mechanisms and structural damage to proteins and DNA [10], which leads to brain tissue damage. Its progression is associated to biochemical changes, like cholinergic deficit, neuronal metabolic damage derived from the glutamate excitotoxicity, and oxidative stress [11]. Acetylcholinesterase inhibitors (AChEI) are actually the best available pharmacotherapy for Alzheimer's disease symptoms treatment, increasing the levels of the acetylcholine neurotransmitter during the cerebral cortex synapses [12].

Flavonoids comprise a large family of plant-derived structurally-related polyphenolic compounds that are classified as anthocyanidins, flavonols, chalcones, aurones, flavanones, isoflavones, flavans, flavanonols, flavanols, and flavones [13]. Flavonoids have been found to display biological activities, such as anti-inflammatory [14], hepatoprotective [15], antiulcer [16], enzyme inhibitors, such as cyclooxygenase [17], xanthine oxidase [18], lipoxygenase [19], phosphodiesterase [20], as well as antiviral [21], cardioprotective [22], anticancer [23], and antioxidant [24] functions.

Pharmacological effects of flavonoids are related to their antioxidant activity, as they are powerful antioxidants against free radicals and ROS [12]. Structurally, the free radical scavenging capacity is primarily attributed to high reactivity of their hydroxyl substituents that participate in the reaction [25]. In this context, we report the chemical synthesis of chalcone and flavone analogs with different substituents on the respective B ring (Figure 1). In addition to the mandatory molecule characterization, their antioxidant and $\mathrm{AChE}$ inhibition activity was evaluated by in vitro testing, to get preliminary insights regarding the structure-activity relationship, allowing for us to further develop the continuous search for new therapeutic agents.

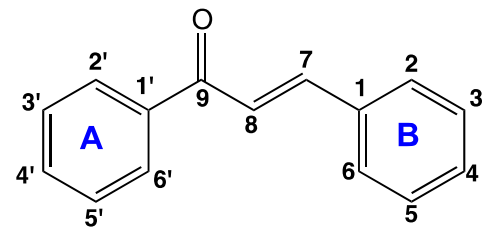

(a)

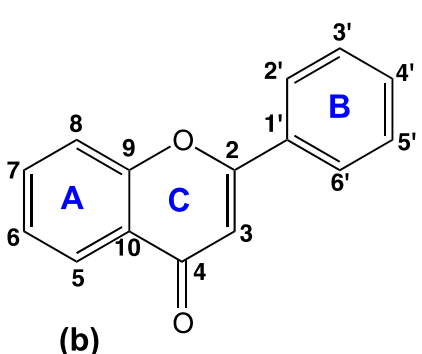

(b)

Figure 1. Basic structure and B ring position of chalcone (a) and flavone (b) molecules.

\section{Materials and Methods}

\subsection{General Information}

All commercial reagents and solvents were used as received and did not require any purification before use. Melting points were taken on a Mel-Temp melting point apparatus (Thermo Scientific). UV spectra were recorded on UV-VIS spectrophotometer model type Genesys 20 and expressed in nm. FT-IR spectroscopic studies were carried out on a FT-IR spectrophotometer Nicolet is5 (Thermo Scientific). NMR spectra were recorded on a Bruker Avance III spectrometer $400 \mathrm{MHz}$. The chemical shifts $(\delta)$ are presented with tetramethylsilane (TMS) $(\delta: 0.00)$ as the internal standard. Gas chromatography-Mass spectrometry data were recorded on a Thermo Scientific TRACE 1310 (GC) 
and Thermo Scientific single quadrupole ISQ LT (MS), with a column model TG-SQC $(30 \mathrm{~m} \times 0.25 \mathrm{~mm}$ inner diameter, $0.25 \mu \mathrm{m}$ film thickness). The detector temperature was $240{ }^{\circ} \mathrm{C}$, the injector temperature was $250^{\circ} \mathrm{C}$, and transfer-line temperature was $250^{\circ} \mathrm{C}$; oven temperature started at $120^{\circ} \mathrm{C}$ for $1 \mathrm{~min}$, increased at a $40{ }^{\circ} \mathrm{C} / \mathrm{min}$ rate until $280{ }^{\circ} \mathrm{C}$, with a hold time of $10 \mathrm{~min}$. Helium was employed as carrier gas, at $1 \mathrm{~mL} / \mathrm{min}$ flow with split ratio 1:20. Column chromatography purifications were carried out on Silica Gel $60 \AA$ (Sigma-Aldrich, 230-400 mesh, Sigma-Aldrich, Milwaukee, WI, USA). The purity of compounds was checked by thin-layer chromatography (TLC) carried out on aluminum backed silica plates by Merck (Kenilworth, NJ, USA) and plates were revealed using a UV $254 \mathrm{~nm}$ light.

\subsection{Synthesis of Chalcone Derivatives}

To a mixture of 2-hydroxyacetophenone (1 Eq) and the appropriate benzaldehyde (1a-1k) (1 Eq) in ethanol/water $(8: 2)(10 \mathrm{~mL})$, was added dropwise aqueous $\mathrm{NaOH} 50 \%(4 \mathrm{~mL})$ at $0{ }^{\circ} \mathrm{C}$. The reaction was stirred at room temperature till completion of reaction (monitored by TLC). The reaction solvent was completely evaporated to redissolve the crude in water and neutralize with a $10 \% \mathrm{HCl}$ solution. The precipitate was filtered, washed with cold water, dried, and purified by column chromatography, employing eluent mixtures of n-hexane/ethyl acetate and dichloromethane/ethyl acetate in different proportions; some products were purified by recrystallization with a methanol $/ \mathrm{H}_{2} \mathrm{O}(1: 2)$ mixture. All of the structures were confirmed by mass and NMR spectra as discussed below.

(E)-1-(2-hydroxyphenyl)-3-phenylprop-2-en-1-one (2a): White solid (yield 46\%). $\quad \mathrm{C}_{15} \mathrm{H}_{12} \mathrm{O}_{2}$. $\mathrm{Mp}=58-60^{\circ} \mathrm{C}$. IR (ATR diamond, $\left.\mathrm{cm}^{-1}\right)=3358(\mathrm{O}-\mathrm{H}), 3031(\mathrm{C}-\mathrm{H}), 1683(\mathrm{C}=\mathrm{O}) \mathrm{cm}^{-1} .{ }^{1} \mathrm{H}$ NMR $\left(400 \mathrm{MHz}, \mathrm{CDCl}_{3}\right) \delta: 12.79(\mathrm{~s}, 1 \mathrm{H}, \mathrm{OH}), 7.93(\mathrm{~d}, J=15.3 \mathrm{~Hz}, 1 \mathrm{H}, \mathrm{H7}), 7.92\left(\mathrm{~d}, J=8.1 \mathrm{~Hz}, 1 \mathrm{H}, \mathrm{H}^{\prime}\right), 7.67$ $(\mathrm{d}, J=15.2 \mathrm{~Hz}, 1 \mathrm{H}, \mathrm{H} 8), 7.50-7.37$ (m, 6H, H2, H3, H4, H5, H6, H4' $), 7.06$ (d, J = 8.6 Hz, 1H, H5'), 6.95 $\left(\mathrm{d}, J=7.3 \mathrm{~Hz}, 1 \mathrm{H}, \mathrm{H3} 3^{\prime}\right) .{ }^{13} \mathrm{C}$ NMR $\left(100 \mathrm{MHz}, \mathrm{CDCl}_{3}\right)$ 8: 193.8 (s, C9), 163.7 (s, C6'), 145.5 (d, C7), 136.5 (d, C4'), 134.7 (s, C1), 131.0 (d, C2'), 129.7 (d, C3, C5), 129.1 (d, C2, C6), 128.7 (d, C4), 126.2 (d, C3'), $120.3\left(\mathrm{~s}, \mathrm{C} 1^{\prime}\right), 118.9$ (d, C8), 118.7 (d, C5'). GC-MS m/z (rel. int.) = $224.15[\mathrm{M}]^{+}$(11), 223.16 (12), 147.08 (17), 120.06 (49), 104.11 (54), 92.05 (100), 77.08 (54).

(E)-1,3-bis(2-hydroxyphenyl)prop-2-en-1-one (2b): Yellow crystals (yield $41 \%$ ). $\quad \mathrm{C}_{15} \mathrm{H}_{12} \mathrm{O}_{3}$. $\mathrm{Mp}=137^{\circ} \mathrm{C}$. IR (ATR diamond, $\left.\mathrm{cm}^{-1}\right)=3267(\mathrm{O}-\mathrm{H}), 3033(\mathrm{C}-\mathrm{H}), 1683(\mathrm{C}=\mathrm{O}), 1150(\mathrm{C}-\mathrm{O}) \mathrm{cm}^{-1}$. ${ }^{1} \mathrm{H}$ NMR $\left(400 \mathrm{MHz}, \mathrm{CDCl}_{3}\right) \delta: 12.63(\mathrm{~s}, 1 \mathrm{H}, \mathrm{OH}), 10.36(\mathrm{~s}, 1 \mathrm{H}, \mathrm{OH}), 8.21(\mathrm{~d}, J=15.3 \mathrm{~Hz}, 1 \mathrm{H}, \mathrm{H7}), 8.21$ $\left(\mathrm{dd}, J_{1}=7.9, J_{2}=1.4 \mathrm{~Hz}, 1 \mathrm{H}, \mathrm{H}^{\prime}\right), 8.00(\mathrm{~d}, J=15.6 \mathrm{~Hz}, 1 \mathrm{H}, \mathrm{H} 8), 7.92\left(\mathrm{dd}, J_{1}=7.8, J_{2}=1.0 \mathrm{~Hz}, 1 \mathrm{H}, \mathrm{H} 6\right)$, $7.58\left(\mathrm{ddd}, J_{1}=8.2, J_{2}=8.6, J_{3}=1.4 \mathrm{~Hz}, 1 \mathrm{H}, \mathrm{H} 4^{\prime}\right), 7.33\left(\mathrm{ddd}, J_{1}=8.2, J_{2}=8.4, J_{3}=1.3 \mathrm{~Hz}, 1 \mathrm{H}, \mathrm{H} 4\right), 7.03$ $\left(\mathrm{ddd}, J_{1}=7.1 \mathrm{~Hz}, 1 \mathrm{H}, \mathrm{H} 5\right), 6.99\left(\mathrm{dd}, J_{1}=8.3 \mathrm{~Hz}, 1 \mathrm{H}, \mathrm{H} 5^{\prime}\right), 6.92\left(\mathrm{dd}, J_{1}=7.5 \mathrm{~Hz}, 1 \mathrm{H}, \mathrm{H} 3^{\prime}\right), 6.02(\mathrm{ddd}$, $\left.J_{1}=8.3 \mathrm{~Hz}, 1 \mathrm{H}, \mathrm{H} 3\right) .{ }^{13} \mathrm{C}$ NMR $\left(100 \mathrm{MHz}, \mathrm{CDCl}_{3}\right) \delta: 193.8$ (s, C9), 157.4 (s, C2), 140.3 (d, C7), 136.0 (d, C4'), $132.4\left(\mathrm{~d}, \mathrm{C2} 2^{\prime}\right), 130.5(\mathrm{~d}, \mathrm{C} 4), 128.9(\mathrm{~d}, \mathrm{C} 6), 121.1(\mathrm{~s}, \mathrm{C} 1), 120.7\left(\mathrm{~d}, \mathrm{C} 3^{\prime}\right), 120.2\left(\mathrm{~s}, \mathrm{C1} 1^{\prime}\right), 119.4(\mathrm{~d}, \mathrm{C} 8)$, $119.0(\mathrm{~d}, \mathrm{C} 5), 117.7\left(\mathrm{~d}, \mathrm{C} 5^{\prime}\right), 116.2(\mathrm{~d}, \mathrm{C} 3)$. GC-MS m/z (rel. int.) $=240.18[\mathrm{M}]^{+}(1), 222.18(8), 147.11(2)$, 121.09 (28), 92.1 (100), 63.07 (73), 39.06 (56).

(E)-3-(4-hydroxy-3-methoxyphenyl)-1-(2-hydroxyphenyl)prop-2-en-1-one (2c): Orange crystals (yield $42 \%$ ). $\mathrm{C}_{16} \mathrm{H}_{14} \mathrm{O}_{4} \cdot \mathrm{Mp}=120^{\circ} \mathrm{C}$. IR (ATR diamond, $\mathrm{cm}^{-1}$ ) = $3377(\mathrm{O}-\mathrm{H}), 3009(\mathrm{C}-\mathrm{H}), 2965(\mathrm{C}-\mathrm{H}), 1682$ $(\mathrm{C}=\mathrm{O}) \mathrm{cm}^{-1} .{ }^{1} \mathrm{H}$ NMR $\left(400 \mathrm{MHz}, \mathrm{CDCl}_{3}\right) \delta: 12.90(\mathrm{~s}, 1 \mathrm{H}, \mathrm{OH}), 7.94\left(\mathrm{dd}, J_{1}=8.1, J_{2}=1.6 \mathrm{~Hz}, 1 \mathrm{H}, \mathrm{H} 2^{\prime}\right)$, $7.93(\mathrm{~d}, J=15.2 \mathrm{~Hz}, 1 \mathrm{H}, \mathrm{H} 7), 7.52(\mathrm{~d}, J=15.6 \mathrm{~Hz}, 1 \mathrm{H}, \mathrm{H} 8), 7.51\left(\mathrm{ddd}, J_{1}=8.4, J_{2}=8.4, J_{3}=1.6 \mathrm{~Hz}, 1 \mathrm{H}\right.$,

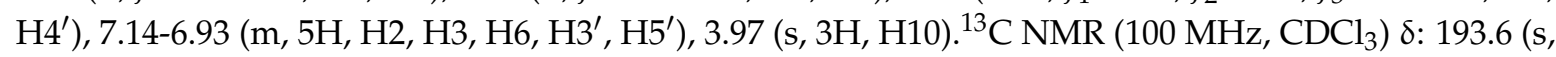
C9), $163.5\left(\mathrm{~s}, \mathrm{C} 6^{\prime}\right), 148.7(\mathrm{~s}, \mathrm{C} 3), 146.9(\mathrm{~s}, \mathrm{C} 4), 145.8(\mathrm{~d}, \mathrm{C} 7), 136.1\left(\mathrm{~d}, \mathrm{C} 4^{\prime}\right), 129.5\left(\mathrm{~d}, \mathrm{C2} 2^{\prime}\right), 127.4(\mathrm{~s}, \mathrm{C} 1)$, $123.7(\mathrm{~d}, \mathrm{C} 6), 121.6\left(\mathrm{~d}, \mathrm{C} 3^{\prime}\right), 120.9\left(\mathrm{~s}, \mathrm{C}^{\prime}\right), 118.7(\mathrm{~d}, \mathrm{C} 8), 118.1\left(\mathrm{~d}, \mathrm{C} 5^{\prime}\right), 117.6(\mathrm{~d}, \mathrm{C} 5), 110.3(\mathrm{~d}, \mathrm{C} 2), 56.1$ $(\mathrm{q}, \mathrm{C} 10)$. GC-MS m/z (rel. int.) = 270.25 [M] ${ }^{+}(4), 150.19$ (28), 135.16 (29), 121.15 (16), 120.15 (26), 107.16 (33), 92.11 (100), 77.15 (68), 64.12 (68).

(E)-3-(2-hydroxy-5-nitrophenyl)-1-(2-hydroxyphenyl)prop-2-en-1-one (2d): Yellow powder (yield 46\%). $\mathrm{C}_{15} \mathrm{H}_{11} \mathrm{NO}_{5} . \mathrm{Mp}=120^{\circ} \mathrm{C}$. IR (ATR diamond, $\mathrm{cm}^{-1}$ ) = $3565(\mathrm{O}-\mathrm{H}), 3068(\mathrm{C}-\mathrm{H}), 1656(\mathrm{C}=\mathrm{O}), 1578(\mathrm{~N}-\mathrm{O})$, 
$1337(\mathrm{~N}-\mathrm{O}), \mathrm{cm}^{-1} .{ }^{1} \mathrm{H}$ NMR $\left(400 \mathrm{MHz}, \mathrm{CDCl}_{3}\right) \delta: 12.44(\mathrm{~s}, 1 \mathrm{H}, \mathrm{OH}), 8.81(\mathrm{~d}, J=2.7 \mathrm{~Hz}, 1 \mathrm{H}, \mathrm{H6}), 8.28$ $\left(\mathrm{dd}, J_{1}=8.4, J_{2}=1.3 \mathrm{~Hz}, 1 \mathrm{H}, \mathrm{H} 4\right), 8.196(\mathrm{~d}, J=15.5 \mathrm{~Hz}, 1 \mathrm{H}, \mathrm{H7}), 8.192\left(\mathrm{dd}, J_{1}=9.2, J_{2}=2.8 \mathrm{~Hz}, 1 \mathrm{H}, \mathrm{H} 2^{\prime}\right)$, $8.10(\mathrm{~d}, J=15.7 \mathrm{~Hz}, 1 \mathrm{H}, \mathrm{H} 8), 7.60\left(\mathrm{dd}, J_{1}=7.4, J_{2}=1.4 \mathrm{~Hz}, 1 \mathrm{H}, \mathrm{H} 4^{\prime}\right), 7.13\left(\mathrm{~d}, J=9.1 \mathrm{~Hz}, 1 \mathrm{H}, \mathrm{H3} 3^{\prime}\right), 7.04$ $\left(\mathrm{dd}, J_{1}=7.8, J_{2}=1.0 \mathrm{~Hz}, 1 \mathrm{H}, \mathrm{H} 5^{\prime}\right), 7.03(\mathrm{~d}, J=7.8 \mathrm{~Hz}, 1 \mathrm{H}, \mathrm{H} 3) .{ }^{13} \mathrm{C} \mathrm{NMR}\left(100 \mathrm{MHz}, \mathrm{CDCl}_{3}\right) \delta: 193.5(\mathrm{~s}$, C9), 162.8 (s, C2), 161.8 (s, C6 '), $140.0(\mathrm{~s}, \mathrm{C} 5), 137.4(\mathrm{~d}, \mathrm{C} 7), 136.2\left(\mathrm{~d}, \mathrm{C4} 4^{\prime}\right), 130.9\left(\mathrm{~d}, \mathrm{C2} 2^{\prime}\right), 127.3$ (d, C4, C6), $124.5\left(\mathrm{~d}, \mathrm{C}^{\prime}\right), 123.2\left(\mathrm{~s}, \mathrm{C1} 1^{\prime}\right), 121.7(\mathrm{~d}, \mathrm{C} 8), 119.0\left(\mathrm{~d}, \mathrm{C}^{\prime}\right), 117.6(\mathrm{~s}, \mathrm{C} 1), 116.6$ (d, C3). GC-MS m/z (rel. int.) = $285.15[\mathrm{M}]^{+}$(1), 253.64 (1), 220.22 (4), 191.54 (3), 171.27 (6), 149.28 (6), 137.01 (16), 117.17 (18), 84.18 (51), 73.10 (32), 69.20 (41), 63.11 (49), 61.14 (41), 30.16 (100).

(E)-1-(2-hydroxyphenyl)-3-(4-methoxyphenyl)prop-2-en-1-one (2e): Yellow crystals (yield 40\%). $\mathrm{C}_{16} \mathrm{H}_{14} \mathrm{O}_{3} . \mathrm{Mp}=76-78{ }^{\circ} \mathrm{C}$. IR (ATR diamond, $\mathrm{cm}^{-1}$ ) = $3082(\mathrm{C}-\mathrm{H}), 2963(\mathrm{C}-\mathrm{H}), 1685(\mathrm{C}=\mathrm{O}), 1255$ $(\mathrm{C}-\mathrm{O}) \mathrm{cm}^{-1} .{ }^{1} \mathrm{H}$ NMR $\left(400 \mathrm{MHz}, \mathrm{CDCl}_{3}\right) \delta: 12.91(\mathrm{~s}, 1 \mathrm{H}, \mathrm{OH}), 7.919\left(\mathrm{dd}, J_{1}=8.0, J_{2}=1.7 \mathrm{~Hz}, 1 \mathrm{H}, \mathrm{H} 2^{\prime}\right)$, $7.911(\mathrm{~d}, J=15.0 \mathrm{~Hz}, 1 \mathrm{H}, \mathrm{H} 7), 7.62\left(\mathrm{dd}, J_{1}=8.8, J_{2}=1.7 \mathrm{~Hz}, 1 \mathrm{H}, \mathrm{H} 2\right), 7.60\left(\mathrm{dd}, J_{1}=8.5, J_{2}=1.7 \mathrm{~Hz}, 1 \mathrm{H}\right.$, H6), $7.54(\mathrm{~d}, J=15.0 \mathrm{~Hz}, 1 \mathrm{H}, \mathrm{H} 8), 7.49\left(\mathrm{ddd}, J_{1}=8.6, J_{2}=8.5, J_{3}=1.5 \mathrm{~Hz}, 1 \mathrm{H}, \mathrm{H} 4^{\prime}\right), 7.02\left(\mathrm{dd}, J_{1}=8.4\right.$, $\left.J_{2}=1.0 \mathrm{~Hz}, 1 \mathrm{H}, \mathrm{H}^{\prime}\right), 6.95\left(\mathrm{dd}, J_{1}=8.8, J_{2}=2.0 \mathrm{~Hz}, 2 \mathrm{H}, \mathrm{H} 3, \mathrm{H} 5\right), 6.94\left(\mathrm{ddd}, J_{1}=9.6, J_{2}=10.9, J_{3}=1.2\right.$ $\left.\mathrm{Hz}, 1 \mathrm{H}, \mathrm{H3} 3^{\prime}\right), 3.85$ (s, 3H, H10). ${ }^{13} \mathrm{C}$ NMR (100 MHz, CDCl 3 ) $\delta: 193.8$ (s, C9), 163.7 (s, C6'), 162.1 (s, C4), 145.4 (d, C7), 136. 2 (s, C4'),130.6 (d, C2, C6), 129.6 (d, C2'), 127.5 (s, C1), 120.2 (s, C1'), 118.8 (d, C5'), 118.7 (d, C3'), 117.7 (d, C8), 114.6 (d, C3, C5), 55.5 (q, C10). GC-MS m/z (rel. int.) = 254.19 [M] (17), 253.20 (12), 134.12 (100), 121.11 (34), 119.12 (40), 92.07 (79), 65.08 (84), 63.07 (90).

(E)-1-(2-hydroxyphenyl)-3-(3-methoxyphenyl)prop-2-en-1-one (2f): Orangish yellow crystals (yield 40\%). $\mathrm{C}_{16} \mathrm{H}_{14} \mathrm{O}_{3} . \mathrm{Mp}=86-90^{\circ} \mathrm{C}$. IR (ATR diamond, $\left.\mathrm{cm}^{-1}\right)=3081(\mathrm{C}-\mathrm{H}), 2918(\mathrm{C}-\mathrm{H}), 1636(\mathrm{C}=\mathrm{O}), 1253$ $(\mathrm{C}-\mathrm{O}) \mathrm{cm}^{-1} .{ }^{1} \mathrm{H}$ NMR $\left(400 \mathrm{MHz}, \mathrm{CDCl}_{3}\right) \delta: 12.77(\mathrm{~s}, 1 \mathrm{H}, \mathrm{OH}), 7.91\left(\mathrm{dd}, J_{1}=8.0, J_{2}=1.6 \mathrm{~Hz}, 1 \mathrm{H}, \mathrm{H}^{\prime}\right)$, $7.89(\mathrm{~d}, J=15.8 \mathrm{~Hz}, 1 \mathrm{H}, \mathrm{H} 7), 7.63(\mathrm{~d}, J=15.4 \mathrm{~Hz}, 1 \mathrm{H}, \mathrm{H} 8), 7.51\left(\mathrm{ddd}, J_{1}=8.5, J_{2}=8.6, J_{3}=1.6 \mathrm{~Hz}, 1 \mathrm{H}\right.$, $\left.\mathrm{H} 4^{\prime}\right), 7.36\left(\mathrm{dd}, J_{1}=7.8 \mathrm{~Hz}, 1 \mathrm{H}, \mathrm{H6}\right), 7.26\left(\mathrm{dd}, J_{1}=7.6 \mathrm{~Hz}, 1 \mathrm{H}, \mathrm{H} 4\right), 7.16\left(\mathrm{dd}, J_{1}=2.2, J_{2}=1.8 \mathrm{~Hz}, 1 \mathrm{H}\right.$, $\mathrm{H} 2), 7.03\left(\mathrm{dd}, J_{1}=8.4, J_{2}=1.0 \mathrm{~Hz}, 1 \mathrm{H}, \mathrm{H} 5^{\prime}\right), 6.99\left(\mathrm{ddd}, J_{1}=8.1, J_{2}=8.2, J_{3}=1.0 \mathrm{~Hz}, 1 \mathrm{H}, \mathrm{H} 5\right), 6.96(\mathrm{ddd}$, $\left.J_{1}=8.1, J_{2}=8.2, J_{3}=1.1 \mathrm{~Hz}, 1 \mathrm{H}, \mathrm{H}^{\prime}\right), 3.85(\mathrm{~s}, 3 \mathrm{H}, \mathrm{H} 10) .{ }^{13} \mathrm{C} \mathrm{NMR}\left(100 \mathrm{MHz}, \mathrm{CDCl}_{3}\right) \delta: 193.85$ (s, C9), $163.7\left(\mathrm{~s}, \mathrm{C} 6^{\prime}\right), 160.1$ (s, C3), 145.5 (d, C7), $136.5\left(\mathrm{~d}, \mathrm{C4} 4^{\prime}\right), 136.13$ (s, C1), 130.1 (d, C2'), 129.8 (d, C5), 121.4 $\left(\mathrm{d}, \mathrm{C} 3^{\prime}\right), 120.6\left(\mathrm{~s}, \mathrm{C1} 1^{\prime}\right), 120.1$ (d, C6), 118.9 (d, C8), 118.7 (d, C5'), 116.7 (d, C4), 113.9 (d, C2), 55.5 (q, C10). GC-MS m/z (rel. int.) = $254.15\left[\mathrm{M}^{+}(11), 253.18\right.$ (8), 165.12 (3), 147.08 (27), 134.10 (17), 121.05 (52), 102.06 (18), 92.05 (36), 89.06 (41), 65.04 (100).

(E)-3-(3,4-dimethoxyphenyl)-1-(2-hydroxyphenyl)prop-2-en-1-one (2g): yellow crystals (yield 57\%). $\mathrm{C}_{17} \mathrm{H}_{16} \mathrm{O}_{4} . \mathrm{Mp}=88-90{ }^{\circ} \mathrm{C}$. IR (ATR diamond, $\mathrm{cm}^{-1}$ ) = $3079(\mathrm{C}-\mathrm{H}), 1694(\mathrm{C}=\mathrm{O}), 1142(\mathrm{C}-\mathrm{O}) \mathrm{cm}^{-1} \cdot{ }^{1} \mathrm{H}$ $\operatorname{NMR}\left(400 \mathrm{MHz}, \mathrm{CDCl}_{3}\right) \delta: 12.90(\mathrm{~s}, 1 \mathrm{H}, \mathrm{OH}), 7.94\left(\mathrm{dd}, J_{1}=8.1, J_{2}=1.8 \mathrm{~Hz}, 1 \mathrm{H}, \mathrm{H2}{ }^{\prime}\right), 7.89(\mathrm{~d}, J=15.4$, $1 \mathrm{H}, \mathrm{H7}), 7.53(\mathrm{~d}, J=15.3 \mathrm{~Hz}, 1 \mathrm{H}, \mathrm{H} 8), 7.50\left(\mathrm{ddd}, J_{1}=8.4, J_{2}=8.5, J_{3}=1.3,1 \mathrm{H}, \mathrm{H} 4^{\prime}\right), 7.27\left(\mathrm{dd}, J_{1}=8.3, J_{2}\right.$ $=2.0 \mathrm{~Hz}, 1 \mathrm{H}, \mathrm{H} 6), 7.17(\mathrm{~d}, 1 \mathrm{H}, \mathrm{H} 2), 7.03\left(\mathrm{ddd}, J_{1}=8.3, J_{2}=8.3, J_{3}=1.0 \mathrm{~Hz}, 1 \mathrm{H}, \mathrm{H} 3^{\prime}\right), 6.95\left(\mathrm{dd}, J_{1}=8.1\right.$, $\left.J_{2}=1.0 \mathrm{~Hz}, 1 \mathrm{H}, \mathrm{H} 5^{\prime}\right), 6.91(\mathrm{~d}, 1 \mathrm{H}, \mathrm{H} 5), 3.96(\mathrm{~s}, 3 \mathrm{H}, \mathrm{H} 10), 3.93(\mathrm{~s}, 3 \mathrm{H}, \mathrm{H} 11) .{ }^{13} \mathrm{C} \mathrm{NMR}\left(100 \mathrm{MHz}, \mathrm{CDCl}_{3}\right)$

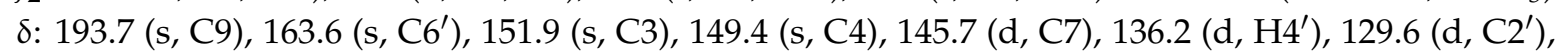
127.7 (s, C1), $123.6(\mathrm{~d}, \mathrm{C} 6), 121.6\left(\mathrm{~d}, \mathrm{C3} 3^{\prime}\right), 120.2\left(\mathrm{~s}, \mathrm{C1} 1^{\prime}\right), 118.8(\mathrm{~d}, \mathrm{C} 8), 117.9\left(\mathrm{~d}, \mathrm{C} 5^{\prime}\right), 111.3(\mathrm{~d}, \mathrm{C} 5), 110.5$ (d, C2), 56.1 (q, C10, C11). GC-MS m/z (rel. int.) = $284.20[\mathrm{M}]^{+}$(8), 165.16 (4), 164.16 (33), 151.15 (25), 121.13 (24), 103.11 (27), 92.06 (69), 77.08 (100).

(E)-3-(4-chlorophenyl)-1-(2-hydroxyphenyl)prop-2-en-1-one (2h): yellow crystals (yield 97\%). $\mathrm{C}_{15} \mathrm{H}_{11} \mathrm{ClO}_{2} . \mathrm{Mp}=70-73^{\circ} \mathrm{C}$. IR (ATR diamond, $\left.\mathrm{cm}^{-1}\right)=3065(\mathrm{C}-\mathrm{H}), 1641(\mathrm{C}=\mathrm{O}), 748(\mathrm{C}-\mathrm{Cl}) \mathrm{cm}^{-1}$. ${ }^{1} \mathrm{H}$ NMR $\left(400 \mathrm{MHz}, \mathrm{CDCl}_{3}\right) \delta: 12.72(\mathrm{~s}, 1 \mathrm{H}, \mathrm{OH}), 7.90\left(\mathrm{dd}, J_{1}=8.0, J_{2}=1.6 \mathrm{~Hz}, 1 \mathrm{H}, \mathrm{H} 2^{\prime}\right), 7.86(\mathrm{~d}$, $J=15.4 \mathrm{~Hz}, 1 \mathrm{H}, \mathrm{H} 7), 7.62(\mathrm{~d}, J=15.4 \mathrm{~Hz}, 1 \mathrm{H}, \mathrm{H} 8), 7.59(\mathrm{~d}, 2 \mathrm{H}, \mathrm{H} 2, \mathrm{H} 6), 7.51\left(\mathrm{ddd}, J_{1}=8.8, J_{2}=8.6\right.$, $\left.J_{3}=1.5 \mathrm{~Hz}, 1 \mathrm{H}, \mathrm{H} 4^{\prime}\right), 7.41(\mathrm{~d}, 2 \mathrm{H}, \mathrm{H} 3, \mathrm{H} 5), 7.03\left(\mathrm{dd}, J_{1}=8.4, J_{2}=1.0 \mathrm{~Hz}, 1 \mathrm{H}, \mathrm{H} 5^{\prime}\right), 6.95\left(\mathrm{ddd}, J_{1}=8.1, J_{2}\right.$

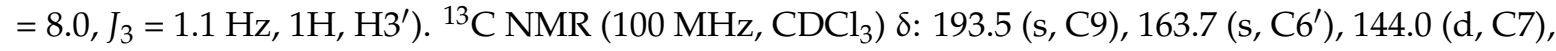
$137.0\left(\mathrm{~d}, \mathrm{C} 4^{\prime}\right), 136.6$ (s, C1), 133.2 (s, C4), 129.9 (d, C2, C6), 129.7 (d, C2'), 129.4 (d, C3, C5), 120.7 (d, 
$\left.\mathrm{C3}^{\prime}\right), 120,0\left(\mathrm{~s}, \mathrm{C1} 1^{\prime}\right), 119.0(\mathrm{~d}, \mathrm{C} 8), 118.8\left(\mathrm{~d}, \mathrm{C}^{\prime}\right)$. GC-MS m/z (rel. int.) = $258.21[\mathrm{M}]^{+}(4), 224.12(5)$, 223.18 (26), 165.18 (7), 147.11 (20), 121.13 (32), 101.10 (39), 93.13 (26), 65.12 (100).

(E)-3-(4-(dimethylamino)phenyl)-1-(2-hydroxyphenyl)prop-2-en-1-one (2i): purple red crystals (yield $51 \%) . \mathrm{C}_{17} \mathrm{H}_{17} \mathrm{NO}_{2} \cdot \mathrm{Mp}=163-165^{\circ} \mathrm{C}$. IR (ATR diamond, $\left.\mathrm{cm}^{-1}\right)=3064(\mathrm{C}-\mathrm{H}), 1661(\mathrm{C}=\mathrm{O}), 1276(\mathrm{C}-\mathrm{N})$ $\mathrm{cm}^{-1} .{ }^{1} \mathrm{H}$ NMR $\left(400 \mathrm{MHz}, \mathrm{CDCl}_{3}\right)$ ): $13.18(\mathrm{~s}, 1 \mathrm{H}, \mathrm{OH}), 7.919(\mathrm{~d}, J=14.9 \mathrm{~Hz}, 1 \mathrm{H}, \mathrm{H} 7), 7.915$ (dd, $\left.J_{1}=8.3, J_{2}=1.8 \mathrm{~Hz}, 1 \mathrm{H}, \mathrm{H} 2^{\prime}\right), 7.56\left(\mathrm{dd}, J_{1}=8.8, J_{2}=1.8 \mathrm{~Hz}, 2 \mathrm{H}, \mathrm{H} 2, \mathrm{H} 6\right), 7.46\left(\mathrm{ddd}, J_{1}=8.5, J_{2}=8.2\right.$, $\left.J_{3}=1.6 \mathrm{~Hz}, 1 \mathrm{H}, \mathrm{H} 4^{\prime}\right), 7.45(\mathrm{~d}, J=15.1 \mathrm{~Hz}, 1 \mathrm{H}, \mathrm{H} 8), 7.00\left(\mathrm{dd}, J_{1}=8.3, J_{2}=1.0 \mathrm{~Hz}, 1 \mathrm{H}, \mathrm{H} 5^{\prime}\right), 6.92(\mathrm{ddd}$, $\left.J_{1}=8.2, J_{2}=8.0, J_{3}=1.2 \mathrm{~Hz}, 1 \mathrm{H}, \mathrm{H3}{ }^{\prime}\right), 6.68\left(\mathrm{dd}, J_{1}=8.9, J_{2}=2.8 \mathrm{~Hz}, 2 \mathrm{H}, \mathrm{H} 3, \mathrm{H} 5\right), 3.03(\mathrm{~s}, 6 \mathrm{H}, \mathrm{H} 10) .{ }^{13} \mathrm{C}$ NMR (100 MHz, CDCl $)$ ) 8: 193.6 (s, C9), 163.6 (s, C6 '), 152.4 (s, C4), 146.6 (d, C7), 135.7 (d, C4'), 130.9 (d, C2, C6), $129.4\left(\mathrm{~d}, \mathrm{C2} 2^{\prime}\right), 122.4(\mathrm{~s}, \mathrm{C} 1), 120.5\left(\mathrm{~d}, \mathrm{C} 3^{\prime}\right), 118.6\left(\mathrm{~s}, \mathrm{C1} 1^{\prime}\right), 118.5(\mathrm{~d}, \mathrm{C} 8), 114.4\left(\mathrm{~d}, \mathrm{C} 5^{\prime}\right), 111.9(\mathrm{~d}$, C3, C5), 40.1 (q, C10). GC-MS m/z (rel. int.) = $267.11[\mathrm{M}]^{+}$(6), 266.11 (3), 220.13 (9), 165.16 (8), 152.16 (3), 104.05 (11), 92.09 (21), 89.06 (39), 76.07 (100).

(E)-3-(anthracen-9-yl)-1-(2-hydroxyphenyl)prop-2-en-1-one (2j): orange crystals (yield 45\%). $\mathrm{C}_{23} \mathrm{H}_{16} \mathrm{O}_{2}$. $\mathrm{Mp}=148-150{ }^{\circ} \mathrm{C}$. IR (ATR diamond, $\left.\mathrm{cm}^{-1}\right)=3046(\mathrm{C}-\mathrm{H}), 1633(\mathrm{C}=\mathrm{O}), 1157(\mathrm{C}-\mathrm{O}) \mathrm{cm}^{-1} .{ }^{1} \mathrm{H}$ NMR $(400$ $\left.\mathrm{MHz}, \mathrm{CDCl}_{3}\right) \delta: 12.85(\mathrm{~s}, 1 \mathrm{H}, \mathrm{OH}), 8.89(\mathrm{~d}, J=15.6 \mathrm{~Hz}, 1 \mathrm{H}, \mathrm{H} 15), 8.45(\mathrm{~s}, 1 \mathrm{H}, \mathrm{H} 8), 8.27\left(\mathrm{dd}, J_{1}=9.4, J_{2}=\right.$ $2.9 \mathrm{~Hz}, 2 \mathrm{H}, \mathrm{H} 3, \mathrm{H} 13), 8.02\left(\mathrm{dd}, J_{1}=9.6, J_{2}=2.9 \mathrm{~Hz}, 2 \mathrm{H}, \mathrm{H} 6, \mathrm{H} 10\right), 7.81\left(\mathrm{dd}, J_{1}=9.6, J_{2}=1.4 \mathrm{~Hz}, 1 \mathrm{H}\right.$, $\left.\mathrm{H} 2^{\prime}\right), 7.65(\mathrm{~d}, J=15.6 \mathrm{~Hz}, 1 \mathrm{H}, \mathrm{H} 16), 7.53-7.46(\mathrm{~m}, 4 \mathrm{H}, \mathrm{H} 4, \mathrm{H} 5, \mathrm{H} 11, \mathrm{H} 12), 7.53$ (ddd, $J_{1}=8.3, J_{2}=8.3, J_{3}$ $\left.=1.7 \mathrm{~Hz}, \mathrm{H} 4^{\prime}\right) 7.08\left(\mathrm{dd}, J_{1}=8.4, J_{2}=1.0 \mathrm{~Hz}, 1 \mathrm{H}, \mathrm{H} 5^{\prime}\right), 6.89\left(\mathrm{ddd}, J_{1}=8.1, J_{2}=8.1, J_{3}=1.1 \mathrm{~Hz}, 1 \mathrm{H}, \mathrm{H} 3^{\prime}\right)$. ${ }^{13} \mathrm{C}$ NMR (100 MHz, CDCl 3 ) 8: 193.3 (s, C17), 163.9 (s, C6 ') 142.7 (d, C15), 136.7 (d, C4'), 131.4 (s, C1), 130.0 (s, C7, C9), 129.8 (d, C6, C10, C2'), 129.5 (d, C3, C13), 129.1 (s, C2, C14), 128.9 (d, C4, C12), 126.7 (d, C5, C11), 125.6 (s, C3'), 120.1 (d, C8, C4'), 119.1 (d, C16), 118.8 (d, C5'). GC-MS m/z (rel. int.) = $324.22[\mathrm{M}]^{+}(27), 281.96$ (5), 268.48 (5), 203.17 (17), 202.14 (35), 200.18 (11), 178.27 (12), 124.79 (15), 121.01 (90), 93.12 (36), 65.05 (100).

(E)-3-(furan-2-yl)-1-(2-hydroxyphenyl)prop-2-en-1-one (2k): yellow crystals (yield 84\%). $\mathrm{C}_{13} \mathrm{H}_{10} \mathrm{O}_{3}$. $\mathrm{Mp}=90-92^{\circ} \mathrm{C}$. IR (ATR diamond, $\mathrm{cm}^{-1}$ ) = 3128-3150 (C-H), $1669(\mathrm{C}=\mathrm{C}), 1636(\mathrm{C}=\mathrm{O}), 1158(\mathrm{C}-\mathrm{O}) \mathrm{cm}^{-1}$. ${ }^{1} \mathrm{H} \mathrm{NMR}\left(400 \mathrm{MHz}, \mathrm{CDCl}_{3}\right) \delta: 12.86(\mathrm{~s}, 1 \mathrm{H}, \mathrm{OH}), 7.91\left(\mathrm{dd}, J_{1}=8.0, J_{2}=1.4 \mathrm{~Hz}, 1 \mathrm{H}, \mathrm{H} 2^{\prime}\right), 7.69(\mathrm{~d}, J=15.1$ $\mathrm{Hz}, 1 \mathrm{H}, \mathrm{H} 5), 7.56(\mathrm{~d}, J=15.1 \mathrm{~Hz}, 1 \mathrm{H}, \mathrm{H6}), 7.55\left(\mathrm{dd}, J_{1}=3.4, J_{2}=1.3 \mathrm{~Hz}, 1 \mathrm{H}, \mathrm{H} 4\right), 7.49$ (ddd, $J_{1}=8.5$, $\left.J_{2}=8.5, J_{3}=1.5 \mathrm{~Hz}, 1 \mathrm{H}, \mathrm{H} 4^{\prime}\right), 6.94\left(\mathrm{ddd}, J_{1}=8.1, J_{2}=8.1, J_{3}=1.0 \mathrm{~Hz}, 1 \mathrm{H}, \mathrm{H} 3^{\prime}\right), 6.76(\mathrm{~d}, 1 \mathrm{H}, \mathrm{H} 2) .{ }^{13} \mathrm{C}$ NMR (100 MHz, CDCl $)_{3}$ ) 8: $193.4(\mathrm{~s}, \mathrm{C} 7), 163.7\left(\mathrm{~s}, \mathrm{C} 6^{\prime}\right), 151.6(\mathrm{~s}, \mathrm{C} 1), 145.5(\mathrm{~d}, \mathrm{C} 4), 136.4\left(\mathrm{~d}, \mathrm{C} 4^{\prime}\right), 131.2$ $\left(\mathrm{d}, \mathrm{C2} 2^{\prime}\right), 129.7(\mathrm{~d}, \mathrm{C} 6), 120.2\left(\mathrm{~d}, \mathrm{C} 3^{\prime}\right), 118.9\left(\mathrm{~s}, \mathrm{C1} 1^{\prime}\right), 118.6(\mathrm{~d}, \mathrm{C} 5), 117.8\left(\mathrm{~d}, \mathrm{C} 5^{\prime}\right), 117.1(\mathrm{~d}, \mathrm{C} 2), 113.0(\mathrm{~d}$, C3). GC-MS m/z (rel. int.) = $214.20[\mathrm{M}]^{+}$(6), 157.12 (3), 131.17 (3), 128.16 (3), 121.12 (23), 94.13 (18), $92.11(13), 77.10(9), 65.11(74), 39.09$ (100).

\subsection{Synthesis of Flavone Derivatives}

To a solution of the corresponding 2-hydroxychalcone (2a-2k) $(1 \mathrm{Eq})$ and $\mathrm{I}_{2}(1 \mathrm{Eq})$, was added DMSO $(5 \mathrm{~mL})$ and the reaction mixture was heated in an oil bath at $130{ }^{\circ} \mathrm{C}$ until the completion of reaction (monitored by TLC). After cooling, the reaction mixture was diluted with water and the iodine was removed by washing with a saturated solution of sodium thiosulfate. The products (3a-3k) were then extracted with ethyl acetate and purified by column chromatography, employing eluent mixtures of n-hexane/ethyl acetate, dichloromethane/ethyl acetate, and ethyl acetate/methanol in different proportions; some products were purified by recrystallization with a methanol/ $\mathrm{H}_{2} \mathrm{O}(1: 2)$ mixture.

2-phenyl-4H-chromen-4-one (3a): white crystals (yield 61\%). $\mathrm{C}_{15} \mathrm{H}_{10} \mathrm{O}_{2} . \mathrm{Mp}=85-87^{\circ} \mathrm{C}$. IR (ATR diamond, $\left.\mathrm{cm}^{-1}\right)=3072(\mathrm{C}-\mathrm{H}), 1639(\mathrm{C}=\mathrm{O}), 1030(\mathrm{C}-\mathrm{O}) \mathrm{cm}^{-1} .{ }^{1} \mathrm{H}$ NMR $\left(400 \mathrm{MHz}, \mathrm{CDCl}_{3}\right)$ 8: $8.25(\mathrm{dd}$, $\left.J_{1}=7.9, J_{2}=1.5 \mathrm{~Hz}, 1 \mathrm{H}, \mathrm{H} 5\right), 7.94\left(\mathrm{dd}, J_{1}=9.6, J_{2}=1.7 \mathrm{~Hz}, 2 \mathrm{H}, \mathrm{H} 2^{\prime}, \mathrm{H} 6^{\prime}\right), 7.72\left(\mathrm{ddd}, J_{1}=8.5, J_{2}=8.6, J_{3}\right.$ $=1.6 \mathrm{~Hz}, 1 \mathrm{H}, \mathrm{H} 7), 7.58-7.50\left(\mathrm{~m}, 4 \mathrm{H}, \mathrm{H} 6, \mathrm{H} 8, \mathrm{H3}^{\prime}, \mathrm{H}^{\prime}\right), 7.44\left(\mathrm{ddd}, J_{1}=8.0, J_{2}=8.0, J_{3}=1.0 \mathrm{~Hz}, 1 \mathrm{H}, \mathrm{H} 4^{\prime}\right)$, $6.82(\mathrm{~s}, 1 \mathrm{H}, \mathrm{H} 3) .{ }^{13} \mathrm{C}$ NMR $\left(100 \mathrm{MHz}, \mathrm{CDCl}_{3}\right)$ 8: 178.5 (s, C4), 163.5 (s, C2), 156.4 (s, C9), 133.8 (d, C7), $131.9\left(\mathrm{~s}, \mathrm{C1} 1^{\prime}\right), 131.7\left(\mathrm{~d}, \mathrm{C} 3^{\prime}\right), 129.1\left(\mathrm{~d}, \mathrm{C} 5^{\prime}\right), 126.4\left(\mathrm{~d}, \mathrm{C} 4^{\prime}\right), 125.8\left(\mathrm{~d}, \mathrm{C} 5, \mathrm{C2}{ }^{\prime}, \mathrm{C} 6^{\prime}\right), 125.3(\mathrm{~s}, \mathrm{C} 10), 124.1(\mathrm{~d}$, 
C6), 118.2 (d, C8), 107.7 (d, C3). GC-MS m/z (rel. int.) = $222.12[\mathrm{M}]^{+}$(19), 221.13 (5), $194.11(14), 165.11$ (7), 120.04 (48), 102.06 (28), 97.07 (15), 92.05 (100).

2-(2-hydroxyphenyl)-4H-chromen-4-one (3b): Pale yellow powder (yield 60\%). $\mathrm{C}_{15} \mathrm{H}_{10} \mathrm{O}_{3} . \mathrm{Mp}=$ 254-256 ${ }^{\circ} \mathrm{C}$. IR (ATR diamond, $\left.\mathrm{cm}^{-1}\right)=3141(\mathrm{O}-\mathrm{H}), 3098(\mathrm{C}-\mathrm{H}), 1638(\mathrm{C}=\mathrm{O}), 1051(\mathrm{C}-\mathrm{O}) \mathrm{cm}^{-1} \cdot{ }^{1} \mathrm{H}$ NMR $\left(400 \mathrm{MHz}, \mathrm{CDCl}_{3}\right) \delta: 11.02(\mathrm{~s}, 1 \mathrm{H}, \mathrm{OH}), 8.17(\mathrm{dd}, 1 \mathrm{H}, \mathrm{H} 5), 8.05\left(\mathrm{ddd}, J_{1}=8.6, J_{2}=8.6, J_{3}=1.1 \mathrm{~Hz}\right.$, $1 \mathrm{H}, \mathrm{H7}), 7.843\left(\mathrm{ddd}, J_{1}=7.8, J_{2}=8.0, J_{3}=1.0 \mathrm{~Hz}, 1 \mathrm{H}, \mathrm{H6}\right), 7.845\left(\mathrm{dd}, J_{1}=8.4, J_{2}=1.4 \mathrm{~Hz}, 1 \mathrm{H}, \mathrm{H} 8\right), 7.80$ $\left(\mathrm{ddd}, J_{1}=8.0, J_{2}=8.0, J_{3}=1.4 \mathrm{~Hz}, 1 \mathrm{H}, \mathrm{H}^{\prime}\right), 7.70\left(\mathrm{dd}, J_{1}=8.6, J_{2}=2.3 \mathrm{~Hz}, 1 \mathrm{H}, \mathrm{H} 6^{\prime}\right), 7.51\left(\mathrm{ddd}, J_{1}=7.9\right.$, $\left.J_{2}=8.0, J_{3}=1.9 \mathrm{~Hz}, 1 \mathrm{H}, \mathrm{H}^{\prime}\right), 7.12(\mathrm{~s}, 1 \mathrm{H}, \mathrm{H} 3), 6.92\left(\mathrm{dd}, 1 \mathrm{H}, \mathrm{H}^{\prime}\right) .{ }^{13} \mathrm{C} \mathrm{NMR}\left(100 \mathrm{MHz}, \mathrm{CDCl}_{3}\right) \delta: 177.0$ (s, C4), 159.1 (s, C2), 156.7 (s, C9), 156.3 (s, C2'), 140.6 (d, C7), 136.2 (d, C4'), 134.1 (d, C6'), 125.2 (d, C5), $124.64(\mathrm{~s}, \mathrm{C} 10), 124.61(\mathrm{~d}, \mathrm{C} 6), 123.0\left(\mathrm{~d}, \mathrm{C}^{\prime}\right), 120.3\left(\mathrm{~s}, \mathrm{C1}{ }^{\prime}\right), 119.5\left(\mathrm{~d}, \mathrm{C} 3^{\prime}\right), 118.5(\mathrm{~d}, \mathrm{C} 8), 111.4(\mathrm{~s}$, C3). GC-MS m/z (rel. int.) = 238.23 [M] $]^{+}(10), 210.20$ (2), 152.27 (3), 121.15 (17), 120.14 (10), 118.16 (12), 92.14 (61), 90.16 (30), 76.15 (21), 64.14 (59), 63.13 (100).

2-(4-hydroxy-3-methoxyphenyl)-4H-chromen-4-one (3c): Yellow powder (yield 40\%). $\mathrm{C}_{16} \mathrm{H}_{12} \mathrm{O}_{4}$. Mp $=198-200{ }^{\circ} \mathrm{C}$. IR (ATR diamond, $\left.\mathrm{cm}^{-1}\right)=3295(\mathrm{O}-\mathrm{H}), 3092(\mathrm{C}-\mathrm{H}), 2935(\mathrm{C}-\mathrm{H}), 1624(\mathrm{C}=\mathrm{O}) \mathrm{cm}^{-1} \cdot{ }^{1} \mathrm{H}$ NMR $\left(400 \mathrm{MHz}, \mathrm{CDCl}_{3}\right) \delta: 8.12\left(\mathrm{dd}, J_{1}=8.0, J_{2}=1.6 \mathrm{~Hz}, 1 \mathrm{H}, \mathrm{H} 5\right), 8.03\left(\mathrm{dd}, J_{2}=2 \mathrm{~Hz}, 1 \mathrm{H}, \mathrm{H} 8\right), 7.82$ $\left(\mathrm{ddd}, J_{1}=8.8, J_{2}=8.4, J_{3}=1.6 \mathrm{~Hz}, 1 \mathrm{H}, \mathrm{H7}\right), 7.74\left(\mathrm{ddd}, J_{1}=8.4, J_{2}=8.4, J_{3}=1 \mathrm{~Hz}, 1 \mathrm{H}, \mathrm{H} 6\right), 7.59(\mathrm{dd}$, $\left.J_{2}=2 \mathrm{~Hz}, 1 \mathrm{H}, \mathrm{H}^{\prime}\right), 7.50\left(\mathrm{dd}, J_{1}=8.8, J_{2}=1.2 \mathrm{~Hz}, 1 \mathrm{H}, \mathrm{H}^{\prime}\right), 6.98\left(\mathrm{~d}, 1 \mathrm{H}, \mathrm{H} 3^{\prime}\right), 6.84(\mathrm{~s}, 1 \mathrm{H}, \mathrm{H} 3), 4.01(\mathrm{~s}$, 3H, H11). ${ }^{13} \mathrm{C}$ NMR (100 MHz, CDCl 3 ) 8: 179.5 (s, C4), 163.9 (s, C2), 157.4 (s, C9), 151.5 (s, C4'), 148.3 (s, $\left.\mathrm{C}^{\prime}\right), 135.3(\mathrm{~d}, \mathrm{C} 7), 130.4(\mathrm{~d}, \mathrm{C} 5), 126.5$ (s, C10), $126.0(\mathrm{~d}, \mathrm{C} 6), 125.4\left(\mathrm{~s}, \mathrm{C1}^{\prime}\right), 124.6\left(\mathrm{~d}, \mathrm{C2}{ }^{\prime}\right), 119.4\left(\mathrm{~d}, \mathrm{C} 3^{\prime}\right)$, 110.4 (d, C8), $106.8\left(\mathrm{~d}, \mathrm{C}^{\prime}\right), 83.7$ (d, C3), 57.0 (q, C11). GC-MS m/z (rel. int.) = $268.24[\mathrm{M}]^{+}(20), 197.16$ (8), 148.18 (16), 121.13 (27), 105.14 (47), 92.12 (95), 63.13 (100).

2-(2-hydroxy-5-nitrophenyl)-4H-chromen-4-one (3d): Pale yellow powder (yield 58\%). $\mathrm{C}_{15} \mathrm{H}_{9} \mathrm{NO}_{5}$. $\mathrm{Mp}=268-270{ }^{\circ} \mathrm{C}$. IR (ATR diamond, $\left.\mathrm{cm}^{-1}\right)=3298(\mathrm{O}-\mathrm{H}), 3024(\mathrm{C}-\mathrm{H}), 1652(\mathrm{C}=\mathrm{O}), 1342(\mathrm{~N}-\mathrm{O}) \mathrm{cm}^{-1}$. GC-MS m/z (rel. int.) = $282.23\left[\mathrm{MM}^{+}\right.$(3), 281.20 (10), 235.18 (3), 179.25 (7), 150.19 (10), 104.12 (9), 92.15 (8), 30.09 (100).

2-(4-methoxyphenyl)-4H-chromen-4-one (3e): white powder (yield 50\%). $\mathrm{C}_{16} \mathrm{H}_{12} \mathrm{O}_{3} . \mathrm{Mp}=158-160{ }^{\circ} \mathrm{C}$. IR (ATR diamond, $\mathrm{cm}^{-1}$ ) = $3051(\mathrm{C}-\mathrm{H}), 2920(\mathrm{C}-\mathrm{H}), 1644(\mathrm{C}=\mathrm{O}), 1024(\mathrm{C}-\mathrm{O}) \mathrm{cm}^{-1} .{ }^{1} \mathrm{H}$ NMR $(400 \mathrm{MHz}$, $\left.\mathrm{CDCl}_{3}\right) \delta: 8.22\left(\mathrm{dd}, J_{1}=7.9, J_{2}=1.5 \mathrm{~Hz}, 1 \mathrm{H}, \mathrm{H} 5\right), 7.87\left(\mathrm{dd}, J_{1}=9 \mathrm{~Hz}, 2 \mathrm{H}, \mathrm{H} 2^{\prime}, \mathrm{H} 6^{\prime}\right), 7.69\left(\mathrm{ddd}, J_{1}=8.5\right.$, $\left.J_{2}=8.7, J_{3}=1.7 \mathrm{~Hz}, 1 \mathrm{H}, \mathrm{H} 7\right), 7.54\left(\mathrm{dd}, J_{1}=8.4 \mathrm{~Hz}, 1 \mathrm{H}, \mathrm{H} 8\right), 7.41\left(\mathrm{ddd}, J_{1}=8.0, J_{2}=8.0, J_{3}=1.0 \mathrm{~Hz}, 1 \mathrm{H}\right.$, $\mathrm{H6}), 7.02\left(\mathrm{dd}, J_{1}=9 \mathrm{~Hz}, 2 \mathrm{H}, \mathrm{H}^{\prime}, \mathrm{H}^{\prime}\right), 6.73(\mathrm{~s}, 1 \mathrm{H}, \mathrm{H} 3), 3.87(\mathrm{~s}, 3 \mathrm{H}, \mathrm{H} 11) .{ }^{13} \mathrm{C} \mathrm{NMR}\left(100 \mathrm{MHz}, \mathrm{CDCl}_{3}\right)$

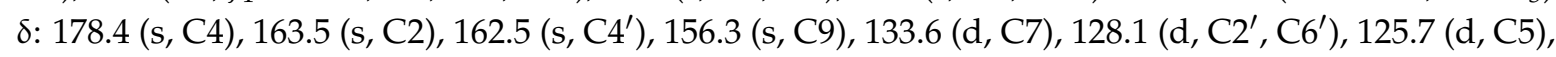
125.1 (d, C6), 124.1 (s, C10), 124.0 (s, C1'), 118.0 (d, C8), 114.5 (d, C3', C5'), 106.3 (d, C3), 55.6 (q, C11). GC-MS m/z (rel. int.) = 252.18 [M] (6), 209.14 (4), 181.09 (2), 132.11 (13), 117.09 (11), 92.05 (64), 89.07 (51), 76.05 (24), 63.07 (100).

2-(3-methoxyphenyl)-4H-chromen-4-one (3f): white powder (yield $49 \%$ ). $\mathrm{C}_{16} \mathrm{H}_{12} \mathrm{O}_{3} . \mathrm{Mp}=118-120{ }^{\circ} \mathrm{C}$. IR (ATR diamond, $\mathrm{cm}^{-1}$ ) = $3036(\mathrm{C}-\mathrm{H}), 2844(\mathrm{C}-\mathrm{H}), 1639(\mathrm{C}=\mathrm{O}), 1130(\mathrm{C}-\mathrm{O}) \mathrm{cm}^{-1} .{ }^{1} \mathrm{H}$ NMR $(400 \mathrm{MHz}$, $\left.\mathrm{CDCl}_{3}\right) \delta: 8.24\left(\mathrm{dd}, J_{1}=8.0, J_{2}=1.2 \mathrm{~Hz}, 1 \mathrm{H}, \mathrm{H} 5\right), 7.71\left(\mathrm{ddd}, J_{1}=8.4, J_{2}=8.8, J_{3}=1.6 \mathrm{~Hz}, 1 \mathrm{H}, \mathrm{H} 5^{\prime}\right), 7.57$ $\left(\mathrm{dd}, J_{1}=8.8 \mathrm{~Hz}, 1 \mathrm{H}, \mathrm{H} 8\right), 7.52\left(\mathrm{ddd}, J_{1}=8.8, J_{2}=8.8, J_{3}=1.2 \mathrm{~Hz}, 1 \mathrm{H}, \mathrm{H7}\right), 7.44\left(\mathrm{ddd}, J_{1}=8.0, J_{2}=2.8\right.$, $\left.J_{3}=2.0 \mathrm{~Hz}, 2 \mathrm{H}, \mathrm{H} 2^{\prime}, \mathrm{H}^{\prime}\right), 7.42\left(\mathrm{dd}, J_{1}=8.0, J_{2}=2.8 \mathrm{~Hz}, 1 \mathrm{H}, \mathrm{H} 4^{\prime}\right), 7.08\left(\mathrm{ddd}, J_{1}=8.4, J_{2}=8.0, J_{3}=1.0\right.$ $\mathrm{Hz}, 1 \mathrm{H}, \mathrm{H6}), 6.83$ (s, 1H, H3), 3.89 (s, 3H, H11). $\left.{ }^{13} \mathrm{C} \mathrm{NMR} \mathrm{(100} \mathrm{MHz,} \mathrm{CDCl}_{3}\right)$ ): 178.6 (s, C4), 163.3 (s, C2), 160.1 (s, C3'), 156.3 (s, C9), $133.9(\mathrm{~d}, \mathrm{C} 7), 133.2\left(\mathrm{~s}, \mathrm{C} 1^{\prime}\right), 130.2\left(\mathrm{~d}, \mathrm{C} 5^{\prime}\right), 125.8(\mathrm{~d}, \mathrm{C} 5), 125.3$ (s, C10), $124.1(\mathrm{~d}, \mathrm{C} 6), 118.8\left(\mathrm{~d}, \mathrm{C}^{\prime}\right), 118.2(\mathrm{~d}, \mathrm{C} 8), 117.3\left(\mathrm{~d}, \mathrm{C4} 4^{\prime}\right), 111.9\left(\mathrm{~d}, \mathrm{C2}^{\prime}\right), 107.9(\mathrm{~d}, \mathrm{C} 3), 55.6(\mathrm{q}, \mathrm{C} 11)$. GC-MS m/z (rel. int.) = 252.25 [M] (32), 251.23 (6), 224.24 (13), 152.19 (12), 132.18 (81), 120.14 (44), 102.17 (63), 92.13 (100). 
2-(3,4-dimethoxyphenyl)-4H-chromen-4-one (3g): white powder (yield 85\%). $\mathrm{C}_{17} \mathrm{H}_{14} \mathrm{O}_{4} \cdot \mathrm{Mp}=$ 128-130 ${ }^{\circ} \mathrm{C}$. IR (ATR diamond, $\mathrm{cm}^{-1}$ ) = $3062(\mathrm{C}-\mathrm{H}), 2929(\mathrm{C}-\mathrm{H}), 1651(\mathrm{C}=\mathrm{O}), 1144(\mathrm{C}-\mathrm{O}) \mathrm{cm}^{-1} .{ }^{1} \mathrm{H}$ NMR $\left(400 \mathrm{MHz}, \mathrm{CDCl}_{3}\right) \delta: 8.23\left(\mathrm{dd}, J_{1}=7.9, J_{2}=1.5 \mathrm{~Hz}, 1 \mathrm{H}, \mathrm{H} 5\right), 7.70\left(\mathrm{ddd}, J_{1}=8.5, J_{2}=8.6, J_{3}=1.7 \mathrm{~Hz}\right.$, $1 \mathrm{H}, \mathrm{H7}), 7.57\left(\mathrm{dd}, J_{1}=8.4, J_{2}=1.9 \mathrm{~Hz}, 2 \mathrm{H}, \mathrm{H} 8, \mathrm{H2}{ }^{\prime}\right), 7.42\left(\mathrm{ddd}, J_{1}=8.0, J_{2}=7.6, J_{3}=1.0 \mathrm{~Hz}, 1 \mathrm{H}, \mathrm{H} 6\right)$, 7.39 (ddd, 1H, H6'), 6.99 (d, 1H, H3'), 6.77 (s, 1H, H3), 3.98 (s, 3H, H11), 3.96 (s, 3H, H12). ${ }^{13} \mathrm{C}$ NMR

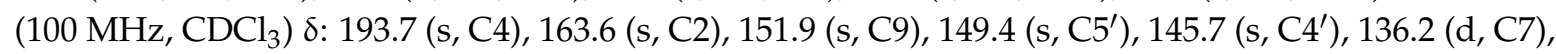
129.6 (d, C5), 127.7 (s, C10), $123.6(\mathrm{~d}, \mathrm{C} 6), 121.6\left(\mathrm{~s}, \mathrm{C1} 1^{\prime}\right), 120.2\left(\mathrm{~d}, \mathrm{C2} 2^{\prime}\right), 118.8(\mathrm{~d}, \mathrm{C} 8), 117.9\left(\mathrm{~d}, \mathrm{C} 3^{\prime}\right), 111.3$ (d, C6 $\left.{ }^{\prime}\right), 110.5$ (d, C3), 56.1 (q, C11, C12). GC-MS m/z (rel. int.) = 282.18 [M] $]^{+}$(35), 168.13 (12), 147.11 (16), 127.18 (25), 121.08 (100), 119.05 (32), 92.07 (86), 91.09 (99), 76.08 (91).

2-(4-chlorophenyl)-4H-chromen-4-one (3h): white powder (yield 82\%). $\mathrm{C}_{15} \mathrm{H}_{9} \mathrm{ClO}_{2} . \mathrm{Mp}=188-190{ }^{\circ} \mathrm{C}$. IR (ATR diamond, $\left.\mathrm{cm}^{-1}\right)=3071(\mathrm{C}-\mathrm{H}), 1647(\mathrm{C}=\mathrm{O}), 756(\mathrm{C}-\mathrm{Cl}) \mathrm{cm}^{-1} .{ }^{1} \mathrm{H}$ NMR $\left(400 \mathrm{MHz}, \mathrm{CDCl}_{3}\right) \delta$ : $8.23\left(\mathrm{dd}, J_{1}=7.9, J_{2}=1.4 \mathrm{~Hz}, 1 \mathrm{H}, \mathrm{H} 5\right), 7.86\left(\mathrm{dd}, J_{1}=8.8 \mathrm{~Hz}, 2 \mathrm{H}, \mathrm{H} 3^{\prime}, \mathrm{H}^{\prime}\right), 7.71\left(\mathrm{ddd}, J_{1}=8.5, J_{2}=8.7\right.$, $\left.J_{3}=1.7 \mathrm{~Hz}, 1 \mathrm{H}, \mathrm{H} 7\right), 7.55\left(\mathrm{dd}, J_{1}=8.4, J_{2}=1.0 \mathrm{~Hz}, 1 \mathrm{H}, \mathrm{H} 8\right), 7.50\left(\mathrm{dd}, J_{1}=8.8 \mathrm{~Hz}, 2 \mathrm{H}, \mathrm{H}^{\prime}{ }^{\prime}, \mathrm{H}^{\prime}\right), 7.43$ $\left(\mathrm{ddd}, J_{1}=8.0, J_{2}=7.8, J_{3}=1.0 \mathrm{~Hz}, 1 \mathrm{H}, \mathrm{H} 6\right), 6.77(\mathrm{~s}, 1 \mathrm{H}, \mathrm{H} 3) .{ }^{13} \mathrm{C} \mathrm{NMR}\left(100 \mathrm{MHz}, \mathrm{CDCl}_{3}\right) \delta: 178.2(\mathrm{~s}$, C4), 162.3 (s, C2), 156.2 (s, C9), $138.0(\mathrm{~d}, \mathrm{C} 7), 134.0\left(\mathrm{~s}, \mathrm{C} 4^{\prime}\right), 130.3\left(\mathrm{~d}, \mathrm{C2}{ }^{\prime}, \mathrm{C} 6^{\prime}\right), 129.4\left(\mathrm{~d}, \mathrm{C} 3^{\prime}, \mathrm{C} 5^{\prime}\right), 127.6$ (s, C1'), 125.8 (d, C5), 125.4 (s, C10), 124.0 (d, C6), 118.1 (d, C8), 107.8 (d, C3). GC-MS m/z (rel. int.) = $258.18[\mathrm{M}]^{+}$(3), 256.18 (10), 228.16 (6), 165.18 (3), 136.13 (13), 120.12 (29), 101.13 (28), 92.11 (100).

2-(4-(dimethylamino)phenyl)-4H-chromen-4-one (3i): white powder (yield 67\%). $\mathrm{C}_{17} \mathrm{H}_{15} \mathrm{NO}_{2}$. $\mathrm{Mp}=$ 193-195 ${ }^{\circ} \mathrm{C}$. IR (ATR diamond, $\left.\mathrm{cm}^{-1}\right)=3057(\mathrm{C}-\mathrm{H}), 1659(\mathrm{C}=\mathrm{O}), 1277(\mathrm{C}-\mathrm{N}) \mathrm{cm}^{-1} .{ }^{1} \mathrm{H}$ NMR $(400 \mathrm{MHz}$, $\left.\mathrm{CDCl}_{3}\right) \delta: 8.17\left(\mathrm{dd}, J_{1}=8.8, J_{2}=2 \mathrm{~Hz}, 1 \mathrm{H}, \mathrm{H} 5\right), 8.04-7.78(\mathrm{~m}, 3 \mathrm{H}, \mathrm{H} 6, \mathrm{H} 7, \mathrm{H} 8), 7.77\left(\mathrm{dd}, 2 \mathrm{H}, \mathrm{H}^{\prime}, \mathrm{H}^{\prime}\right)$, 6.94 (dd, 2H, H2', $\left.\mathrm{H6}^{\prime}\right), 6.84$ (s, 1H, H3), 2.99 (s, 6H, H11). ${ }^{13} \mathrm{C}$ NMR (100 MHz, $\left.\mathrm{CDCl}_{3}\right)$ 8: 176.63 (s, C4), 162.4 (s, C2), 155.4 (s, C9), $152.5\left(\mathrm{~s}, \mathrm{C4} 4^{\prime}\right), 135.7$ (d, C7), 133.8 (d, C2'), 133.5 (d, C6'), 133.1 (d, C5), 125.2 (s, C10), 124.6 (d, C6), $118.2\left(\mathrm{~s}, \mathrm{C} 1^{\prime}\right), 117.9$ (d, C8), 116.7 (d, C3'), 103.9 (d, C3). GC-MS m/z (rel. int. $)=265.26[\mathrm{M}]^{+}(10), 261.68(1), 195.10(2), 104.19(10), 90.71(9), 74.87$ (11), 73.05 (100).

2-(anthracen-9-yl)-4H-chromen-4-one (3j): yellow powder (yield 84\%). $\mathrm{C}_{23} \mathrm{H}_{14} \mathrm{O}_{2} . \mathrm{Mp}=193-195{ }^{\circ} \mathrm{C}$. IR (ATR diamond, $\left.\mathrm{cm}^{-1}\right)=3063(\mathrm{C}-\mathrm{H}), 1643(\mathrm{C}=\mathrm{O}), 1122(\mathrm{C}-\mathrm{O}) \mathrm{cm}^{-1} .{ }^{1} \mathrm{H}$ NMR $\left(400 \mathrm{MHz}, \mathrm{CDCl}_{3}\right) \delta$ : $8.62\left(\mathrm{~s}, 1 \mathrm{H}, \mathrm{H} 8^{\prime}\right), 8.40\left(\mathrm{dd}, J_{1}=7.96, J_{2}=1.6 \mathrm{~Hz}, 1 \mathrm{H}, \mathrm{H} 5\right), 8.08\left(\mathrm{dd}, J_{1}=6.7, J_{2}=1.5 \mathrm{~Hz}, 2 \mathrm{H}, \mathrm{H}^{\prime}, \mathrm{H}^{\prime}\right)$, $7.97\left(\mathrm{dd}, J_{1}=7.9, J_{2}=1.9 \mathrm{~Hz}, 2 \mathrm{H}, \mathrm{H} 10^{\prime}, \mathrm{H}^{\prime} 3^{\prime}\right), 7.73\left(\mathrm{ddd}, J_{1}=8.5, J_{2}=8.6, J_{3}=1.6 \mathrm{~Hz}, 1 \mathrm{H}, \mathrm{H7}\right), 7.53-7.47$ $\left(\mathrm{m}, 6 \mathrm{H}, \mathrm{H6}, \mathrm{H} 8, \mathrm{H}^{\prime}, \mathrm{H}^{\prime}, \mathrm{H} 11^{\prime}, \mathrm{H} 12^{\prime}\right), 6.69(\mathrm{~s}, 1 \mathrm{H}, \mathrm{H} 3) .{ }^{13} \mathrm{C} \mathrm{NMR}\left(100 \mathrm{MHz}, \mathrm{CDCl}_{3}\right)$ 8: 178.1 (s, C4), 163.8 (s, C2), 157.4 (s, C9), $144.0(\mathrm{~d}, \mathrm{C} 7), 134.0\left(\mathrm{~s}, \mathrm{C} 1^{\prime}\right), 131.1\left(\mathrm{~s}, \mathrm{C} 7^{\prime}, \mathrm{C} 9^{\prime}\right), 130.2\left(\mathrm{~d}, \mathrm{C} 6^{\prime}, \mathrm{C} 10^{\prime}\right), 130.0(\mathrm{~d}$, C5), $128.8\left(\mathrm{~s}, \mathrm{C} 2^{\prime}, \mathrm{C} 14^{\prime}\right), 127.3\left(\mathrm{~d}, \mathrm{C} 3^{\prime}, \mathrm{C} 13^{\prime}\right), 126.1\left(\mathrm{~d}, \mathrm{C} 4^{\prime}, \mathrm{C} 12^{\prime}\right), 125.7\left(\mathrm{~d}, \mathrm{C} 5^{\prime}, \mathrm{C} 11^{\prime}\right), 125.6(\mathrm{~s}, \mathrm{C} 10)$, $125.0(\mathrm{~d}, \mathrm{C} 6), 124.3\left(\mathrm{~d}, \mathrm{C} 8^{\prime}\right), 118.5$ (d, C8), 116.3 (d, C3). GC-MS m/z (rel. int.) = $322.27[\mathrm{M}]^{+}(7), 321.25$ (4), 305.25 (9), 202.15 (19), 200.16 (9), 189.11 (2), 120.06 (15), 92.08 (100).

2-(furan-2-yl)-4H-chromen-4-one (3k): brown yellow powder (yield $45 \%$ ). $\mathrm{C}_{13} \mathrm{H}_{8} \mathrm{O}_{3} . \mathrm{Mp}=118-120{ }^{\circ} \mathrm{C}$. IR (ATR diamond, $\mathrm{cm}^{-1}$ ) = $3105(\mathrm{C}-\mathrm{H}), 1651(\mathrm{C}=\mathrm{O}), 1054(\mathrm{C}-\mathrm{O}) \mathrm{cm}^{-1} .{ }^{1} \mathrm{H}$ NMR $\left(400 \mathrm{MHz}, \mathrm{CDCl}_{3}\right) \delta$ : $8.21\left(\mathrm{dd}, J_{1}=7.9, J_{2}=1.5 \mathrm{~Hz}, 1 \mathrm{H}, \mathrm{H} 5\right), 7.68\left(\mathrm{ddd}, J_{1}=8.5, J_{2}=8.7, J_{3}=1.6 \mathrm{~Hz}, 1 \mathrm{H}, \mathrm{H} 7\right), 7.62\left(\mathrm{dd}, J_{1}=2.3\right.$, $\left.J_{2}=1.6 \mathrm{~Hz}, 1 \mathrm{H}, \mathrm{H} 4^{\prime}\right), 7.49\left(\mathrm{dd}, J_{1}=8.4, J_{2}=1.0 \mathrm{~Hz}, 1 \mathrm{H}, \mathrm{H} 8\right), 7.41\left(\mathrm{ddd}, J_{1}=8.0, J_{2}=8.0, J_{3}=1.0 \mathrm{~Hz}, 1 \mathrm{H}\right.$, H6), $7.13\left(\mathrm{~d}, 1 \mathrm{H}, \mathrm{H2}{ }^{\prime}\right), 6.72(\mathrm{~s}, 1 \mathrm{H}, \mathrm{H} 3), 6.60\left(\mathrm{dd}, J_{1}=3.5, J_{2}=1.7 \mathrm{~Hz}, 1 \mathrm{H}, \mathrm{H} 3^{\prime}\right) .{ }^{13} \mathrm{C} \mathrm{NMR}(100 \mathrm{MHz}$,

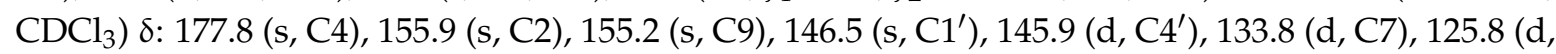
C5), 125.2 (s, C10), 124.3 (d, C6), $117.9(\mathrm{~d}, \mathrm{C} 8), 113.1\left(\mathrm{~d}, \mathrm{C}^{\prime}\right), 112.6(\mathrm{~d}, \mathrm{C} 3), 105.6\left(\mathrm{~d}, \mathrm{C2} 2^{\prime}\right)$. GC-MS m/z $($ rel. int. $)=212.12[\mathrm{M}]^{+}(11), 184.12(7), 156.12(2), 128.12$ (9), 120.07 (24), 92.07 (100), 63.07 (94).

\subsection{In Vitro Antioxidant Activity assay}

\subsubsection{DPPH Radical-Scavenging Assay}

The radical-scavenging activity was performed, as described by Salazar-Aranda et al. [26], with slight modifications. Different concentrations of the sample were prepared with methanol by serial 
dilutions. An aliquot of each dilution $(0.5 \mathrm{~mL})$ was mixed with $0.5 \mathrm{~mL}$ of a methanolic solution of 1,1-diphenyl-2-picrylhydrazyl (DPPH) $(7.5 \mathrm{mg} / 250 \mathrm{~mL})$. The mixtures were incubated at room temperature in the dark for $30 \mathrm{~min}$. Using methanol as a blank, the spectrophotometric measurement was performed at $517 \mathrm{~nm}\left(\mathrm{~A}_{517}\right)$. The radical-scavenging activity was calculated as a percentage of DPPH decoloration using the following formula:

$$
\mathrm{DPPH}(\%)=[1-(\mathrm{B} / \mathrm{A})] \times 100
$$

Where A represents the A value of the control (DPPH solution) and B represents the sample. All of the determinations were performed in triplicate. For each compound, the percentage of DPPH decoloration was plotted against the concentration of each dilution. The concentration that is required to decrease the absorbance of DPPH by $50 \%$ was obtained by interpolation, from a linear regression analysis, and expresses the $\mathrm{EC}_{50}$ value. Quercetin was used as a reference compound.

\subsubsection{ABTS (2-2'-azino-bis-(3-ethylbenzothiazoline-6-sulfonate)) Radical-Scavenging Assay}

ABTS radical cation $\left(\mathrm{ABTS}^{+}\right)$scavenging assay was determined using the methodology developed by Re et al. and Kuskoski et al. $[27,28]$ with slight modifications. ABTS was dissolved in water at $7 \mathrm{mM}$ concentration. ABTS radical cation $\left(\mathrm{ABTS}^{+}\right.$) was produced by reacting ABTS stock solution with $2.45 \mathrm{mM}$ potassium persulfate and then the mixture was kept in the dark at room temperature for 16-18 $\mathrm{h}$ before use. The $\mathrm{ABTS}^{+}$solution $(150 \mu \mathrm{L})$ was diluted with methanol to obtain an absorbance at $0.7 \pm 0.02$, which is taken as initial absorbance. Of this solution, $980 \mu \mathrm{L}$ was added to $20 \mu \mathrm{L}$ of different concentrations of the sample. The mixture was stirred, incubated at room temperature for $30 \mathrm{~min}$, and read at a wavelength of $754 \mathrm{~nm}$, which was taken as the final absorbance. The results are shown as a percentage of inhibition using the following formula:

$$
\% \text { of inhibition }=[(\mathrm{A} 1-\mathrm{A} 2) / \mathrm{A} 1] \times 100
$$

Where A1 is the initial absorbance of the ABTS solution and A2 is the final absorbance of the ABTS solution in the presence of the sample. All of the determinations were performed in triplicate. For each compound, the percentage of inhibition was plotted against the concentration of each dilution. The concentration required to decrease the absorbance of ABTS by $50 \%$ was obtained by interpolation, from a linear regression analysis, and expresses the $\mathrm{EC}_{50}$ value. Quercetin was used as a reference compound.

\subsection{3. $\beta$-Carotene/linoleic Acid bleaching Assay}

Heat-induced oxidation of an aqueous emulsion system of $\beta$-carotene and linoleic acid was used for testing the antioxidant activity that was described by Burda and Oleszek [29], with slight modifications. To $1 \mathrm{~mL}$ of $\beta$-carotene solution $(0.2 \mathrm{mg} / \mathrm{mL}$ in chloroform) was added $20 \mu \mathrm{L}$ linoleic acid and $200 \mu \mathrm{L}$ of Tween 20 . Once the chloroform was evaporated, $50 \mathrm{~mL}$ of distilled water saturated for $1 \mathrm{~h}$ with oxygen was added. An amount of $2.5 \mathrm{~mL}$ of the $\beta$-carotene emulsion was mixed with $0.35 \mathrm{~mL}$ of the corresponding compound solution in methanol $(0.5 \mathrm{mg} / \mathrm{mL})$ and the resulting mixture was shaken. The absorbance of the samples was measured on a spectrometer at $470 \mathrm{~nm}$ immediately after their preparation $(t=0 \mathrm{~min})$, and at the end of the experiment $(t=120 \mathrm{~min})$, during this time the samples were kept at $50{ }^{\circ} \mathrm{C}$. The antioxidant activity (AA) was expressed as percentage of inhibition of $\beta$-carotene bleaching, as compared to the control, and calculated using the following formula:

$$
\mathrm{AA}(\%)=\{1-[(\mathrm{AS} 0-\mathrm{AS} 120) /(\mathrm{AC} 0-\mathrm{AC} 120)]\} \times 100
$$

Where $\mathrm{As}^{0}$ is the absorbance of the sample at $0 \mathrm{~min}, \mathrm{As}^{120}$ is the absorbance of the sample at $120 \mathrm{~min}, \mathrm{Ac}^{0}$ is the absorbance of the control sample at $0 \mathrm{~min}$, and $\mathrm{Ac}^{120}$ is the absorbance of the control sample at $120 \mathrm{~min}$. $\alpha$-tocopherol was used as a reference compound. 


\subsection{In Vitro Acetylcholinesterase Inhibitory Assay}

Acetylcholinesterase activity was determined with the methodology that was reported by Adewusi (2011) [30]. In a 96-well plate was added $75 \mu \mathrm{L}$ of Trizma- $\mathrm{HCl}$ buffer $(50 \mathrm{mM}, \mathrm{pH} 8)$ and $75 \mu \mathrm{L}$ of synthesized compound and serial dilutions were made (final well concentrations were between 150 and $2.4 \mu \mathrm{g} / \mathrm{mL}$ in the buffer, and DMSO at a final maximum concentration of $0.15 \%$ ). Afterwards, to each well was added $25 \mu \mathrm{L}$ of a buffer solution of ATCl $15 \mathrm{mM}$ (final concentration of $1.5 \mathrm{mM}$ ) and $125 \mu \mathrm{L}$ of a buffer solution of DTNB $3 \mathrm{mM}$ (final concentration $1.5 \mathrm{mM}$ ). Absorbance was measured in a microplate reader (Multiskan FC, Thermo Scientific) at $405 \mathrm{~nm}$ every $45 \mathrm{~s}$ in three consecutive times. After that, to each well was added $25 \mu \mathrm{L}$ of an acetylcholinesterase enzyme buffer solution at $2 \mathrm{U} / \mathrm{mL}$ enriched with bovine serum albumin $0.1 \mathrm{mg} / \mathrm{mL}$ (final well enzyme concentration $0.2 \mathrm{U} / \mathrm{mL}$ ) and the absorbance was measured five consecutive times every $45 \mathrm{~s}$. Six wells of each plate were employed without a compound to test, to serve as a $100 \%$ enzymatic activity control. The increase of absorbance due to spontaneous substrate hydrolysis was corrected subtracting the absorbance before the enzyme addition from the absorbance that was obtained after the enzyme addition. Inhibition percentage was calculated by the following equation

$$
\text { inhibition } \%=1-(\text { Asample } / \text { Acontrol }) \times 100
$$

Where $\mathrm{A}_{\text {sample }}$ is the absorbance difference between time 0 and $225 \mathrm{~s}$ in presence of any compound to test or inhibitor, and $A_{\text {control }}$ is the absorbance difference between time 0 and $225 \mathrm{~s}$ of the $100 \%$ enzymatic activity control. Inhibitory concentration $50\left(\mathrm{IC}_{50}\right)$ was calculated by interpolation from the graphic of inhibition percentages in function of the employed concentrations. All of the experiments were performed by triplicate and galantamine was employed as a positive control, which is a drug that is currently used for Alzheimer disease treatment. [12]

\subsection{Molecular Docking}

The three-dimensional structures of the ligands were generated with UCSF Chimera 1.11.2 [31] through their SMILES string. The structures were energy-minimized using Chimera default parameters (which include MMTK, Amber, and Amber's Antechamber parameters) [32]. Rotatable bonds and atomic charges from the ligands were defined with AutoDock Tools 1.5.6. [33]. The crystallographic structure of the AChE receptor (PDB ID:1EVE) in complex with donepezil was downloaded from the Protein Data Bank (https:/ / www.rcsb.org/). The preparation for the receptor with AutoDock Tools included remotion of the co-crystalized ligand and water molecules, hydrogen addition and charges calculation. For the docking, AutoDock Vina [34] was employed, with a grid box of $30 \times 30 \times 30 \AA$ with center coordinates $x=2.27731, y=63.7499$ and $z=65.4998$. Five docking runs with 10 poses each were made per ligand, with an exhaustiveness of 8 . Visualizations of the docked poses for their analysis were made with Chimera. To validate the docking protocol, a re-docking of co-crystallized donepezil was made and the resulting pose was compared to the crystallographic one through their RMSD.

\section{Results and Discussion}

\subsection{Synthesis of Chalcone and Flavone Analogs ( $2 a-2 k, 3 a-3 k)$}

Chalcones are usually synthesized by condensation reactions with acid or basic catalysis, even though recently a great number of new procedures for the synthesis of these molecules due to the great interest on their biological properties have appeared. Claisen-Schmidt reaction with basic conditions is the most widely synthesis reported in the literature, because of the easily process and efficiency in the product formation [35]. The substituted 2-hydroxychalcones $\mathbf{2 a - 2 k}$ were prepared by the Claisen-Schmidt condensation of the respective substituted benzaldehyde $\mathbf{1 a - 1 k}$ (1 eq) and 2-hydroxyacetophenone (1 eq) in the presence of $\mathrm{NaOH}$ in ethanol/water at room temperature by the known literature method (Scheme 1) [36]. This methodology allowed for the obtention of 
eleven compounds with moderate to high yields, between $40-97 \%$, being the highest one the yield for compound $\mathbf{2 h}$. The oxidative cyclization of 2 -hydroxychalcones $\mathbf{2 a} \mathbf{a}-\mathbf{2} \mathbf{k}$ to flavones $\mathbf{3 a} \mathbf{a} \mathbf{3} \mathbf{k}$ was performed using the classical iodine (1 mmol) in DMSO (Scheme 1) system. This method is particularly useful in flavone synthesis starting from 2-hydroxychalcones as it has been observed the iodine does not give secondary reactions despite the high temperature employed [37]. The corresponding flavones were successfully synthesized with yields between $40-85 \%$.<smiles>[R]c1cc(C=O)c([R])c([R2])c1[R]</smiles>

(1a-1i)

1a $R_{1}=R_{2}=R_{3}=R_{4}=H$

1b $\mathrm{R}_{1}=\mathrm{OH}, \mathrm{R}_{2}=\mathrm{R}_{3}=\mathrm{R}_{4}=\mathrm{H}$

1c $\mathrm{R}_{1}=\mathrm{R}_{4}=\mathrm{H}, \mathrm{R}_{2}=\mathrm{OMe}, \mathrm{R}_{3}=\mathrm{OH}$

1d $R_{1}=O H, R_{2}=R_{3}=H, R_{4}=N_{2}$

1e $R_{1}=R_{2}=R_{4}=H, R_{3}=O M e$

If $R_{1}=R_{3}=R_{4}=H, R_{2}=O M e$

1g $\mathrm{R}_{1}=\mathrm{R}_{4}=\mathrm{H}, \mathrm{R}_{2}=\mathrm{R}_{3}=\mathrm{OMe}$

1h $\mathrm{R}_{1}=\mathrm{R}_{2}=\mathrm{R}_{4}=\mathrm{H}, \mathrm{R}_{3}=\mathrm{Cl}$

1i $R_{1}=R_{2}=R_{4}=H, R_{3}=N\left(C_{3}\right)_{2}$

1j 9-anthraldehyde

1k Furfuraldehyde<smiles>[R9]c1cc(/C=C/C(=O)c2ccccc2O)c([R])c([R9])c1[2H]</smiles>

2a-2k

3a-3k

Scheme 1. Reaction Conditions: (i) $\mathrm{NaOH} 50 \%$ /ethanol $-\mathrm{H}_{2} \mathrm{O}$, room temperature, $12 \mathrm{~h}$. (ii) $\mathrm{I}_{2}-\mathrm{DMSO}$, reflux $130{ }^{\circ} \mathrm{C}, 30 \mathrm{~min}$.

All of the compounds were characterized by $\mathrm{IR},{ }^{1} \mathrm{H}$, and ${ }^{13} \mathrm{C}-\mathrm{NMR}$ and mass spectroscopy; in the ${ }^{1} \mathrm{H}$ NMR spectra of the compounds $\mathbf{2 a}-\mathbf{k}$ can be noticed the hydroxyl proton in ring A between $\delta 12.63-13.18 \mathrm{ppm}$, due to the intramolecular hydrogen bond made with the carbonyl oxygen atom. The vinylic protons of the $\alpha, \beta$-unsaturated system are present as doublets for $\mathrm{H}_{\alpha}=7.48-8.10 \mathrm{ppm}$ and $\mathrm{H}_{\beta}=7.69-8.89 \mathrm{ppm}$, being the coupling constants $J \mathrm{H}_{\alpha}-\mathrm{JH}_{\beta}=14.9-15.8 \mathrm{~Hz}$, which indicates a trans configuration for these protons. All of the aromatic protons were observed at their expected shifts, so as their coupling constants. In ${ }^{13} \mathrm{C}-\mathrm{NMR}$ spectra, the carbonyl carbon is observed at $\delta 193.3-193.8 \mathrm{ppm}$, being the $\alpha$ - and $\beta$-carbons at 117.7-121.7 ppm and 129.7-145.8 ppm, respectively.

For the flavone type compounds 3a-k, carbonyl $\alpha$-proton $\mathrm{H}-3$ is observed as a singlet with shifts of 6.69-7.12 ppm, the aromatic protons of the A and B rings were at their expected shifts and coupling constants. Regarding ${ }^{13} \mathrm{C}-\mathrm{NMR}$, the carbonyl carbon shifts to a lower frequency with $\delta$ 177.0-193.7 ppm, being the carbons C-2 and C-3 identified at 155.9-163.9 ppm and 104.5-116.3 ppm, respectively. The assignments were made by HSQC and HMBC experiments.

NMR spectra, including bidimensional ones, of selected chalcones and flavones can be observed in Figures S1-S24 in the supplementary materials.

\subsection{Antioxidant Activity}

To determine antioxidant activities of 2-hydroxychalcones $\mathbf{2 a}-\mathbf{2 k}$ and flavone analogs $\mathbf{3 a}-\mathbf{3 k}$, heat-induced oxidation in a $\beta$-carotene and linoleic acid system, DPPH radical scavenging activity and ABTS radical cation decolorization assay were evaluated. The $\mathrm{EC}_{50}$ values of compounds, quercetin and $\alpha$-tocopherol obtained are shown in Table 1 and Figure 2. 
Table 1. Antioxidant activity of synthesized compounds $2 \mathbf{a}-\mathbf{2 k}$ and $\mathbf{3 a}-\mathbf{3 k}$.

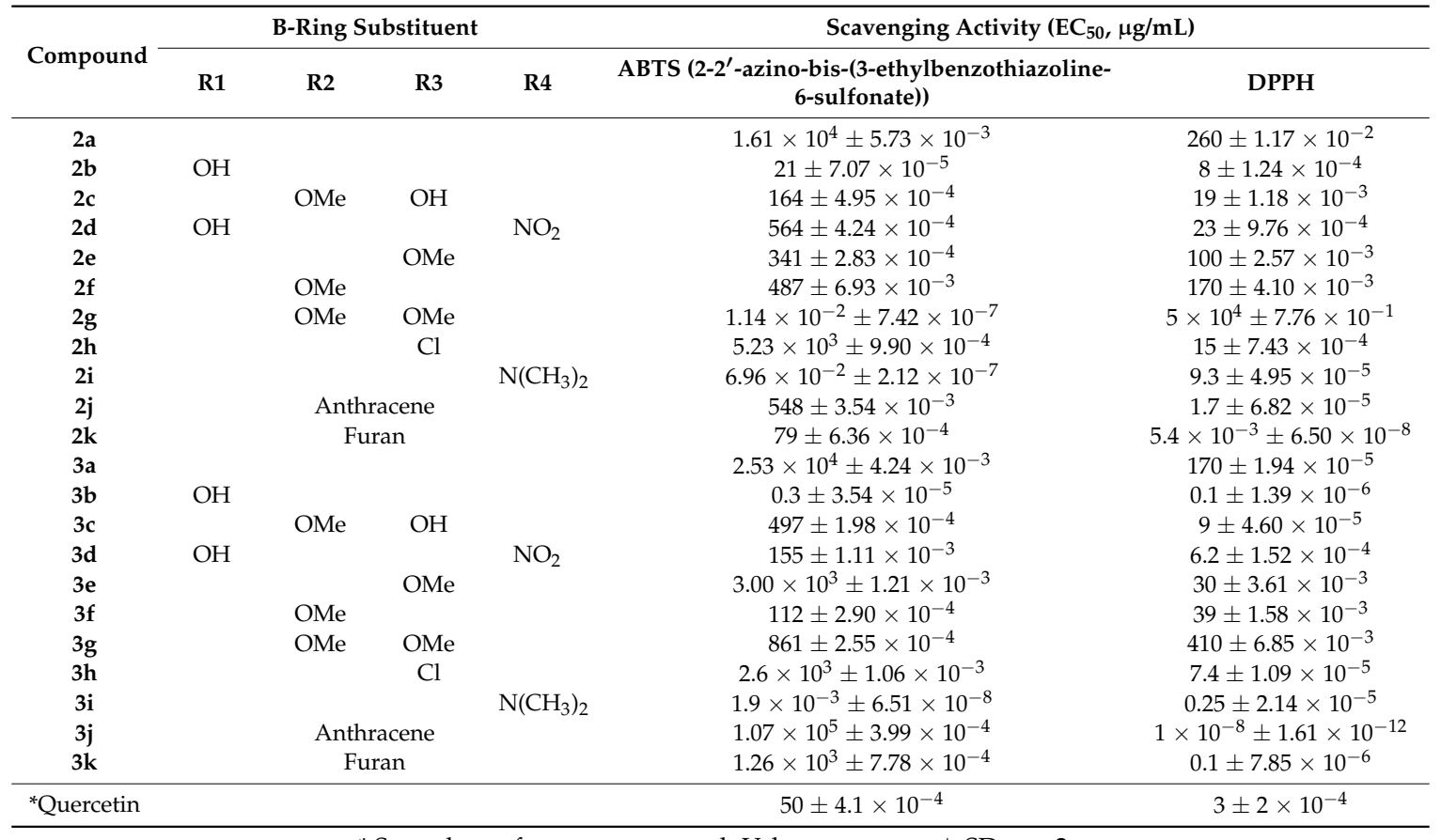

* Served as reference compound. Values are mean $\pm \mathrm{SD}, \mathrm{n}=2$.

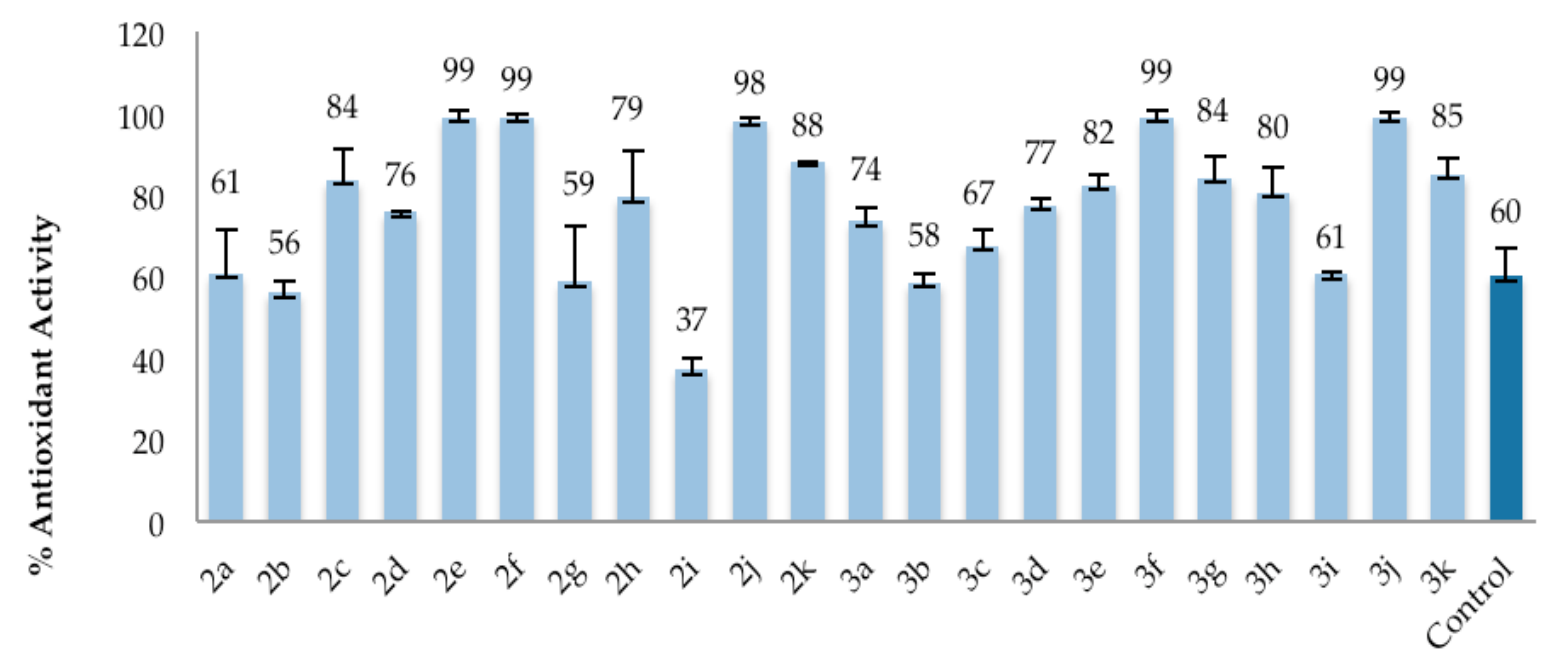

Figure 2. Percentage of antioxidant activity in $\beta$-carotene/linoleic acid bleaching assay of chalcone (2a-2k) and flavone analogs (3a-3k). $\alpha$-tocopherol served as reference compound. Bars are mean $\pm \mathrm{SD}$, $\mathrm{n}=2$.

The results showed that most of the compounds exhibited high activity with DPPH and $\beta$-carotene assay and weak activity in ABTS; additionally, the flavone-type derivatives showed a better antiradical and antioxidant activity as compared to their chalcone precursors. Structure-antioxidant activity relationships of chalcones and flavones suggest this activity is related to a series of different mechanisms, like free radical neutralization, hydrogen donation, singlet oxygen quenching, and metal chelation [38].

The high double bond conjugation of chalcone and flavonoid systems, just as the presence of a C2-C3 double bond and a carbonyl in C-4 position, have been reported as structural characteristics for the antioxidant activity [39], being these incorporated in the basic skeleton of all the synthesized compounds. 
Bearing this in mind, molecules $\mathbf{2 a}$ and $3 \mathbf{a}$ have no substituents in their structures, having the lowest activity of all the generated analogs, therefore it is possible to infer that the addition of at least one substitution favors the antioxidant potential.

In chalcone type molecules, the antioxidant properties are vastly influenced by the two aryl groups and their substitution patterns. The presence of hydroxyl groups to improve the antioxidant activity due to its easy conversion to phenoxy radicals by the hydrogen transfer mechanism is considered to be indispensable [40].

Hydroxyl group presence was evaluated in the $\mathbf{2} \mathbf{b}(o-\mathrm{OH}), \mathbf{2} \mathbf{c}(p-\mathrm{OH}, m-\mathrm{OMe})$, and $\mathbf{2} \mathbf{d}(o-\mathrm{OH}$, $m-\mathrm{NO}_{2}$ ) analogs; despite that the three had great activity in the tested techniques (see Table 1$), \mathbf{2} \mathbf{b}$ showed better results when compared to the other analogs in the antiradical techniques DPPH and ABTS, with an $\mathrm{EC}_{50}$ of $8 \mu \mathrm{g} / \mathrm{mL}$ and $21 \mu \mathrm{g} / \mathrm{mL}$ respectively, surpassing quercetin as the control used in ABTS $\left(\mathrm{EC}_{50}\right.$ of $50 \mu \mathrm{g} / \mathrm{mL}$ ). This indicates that in the radical reduction mechanism by hydrogen donation (like DPPH and ABTS), the addition of other substitutions different from hydroxyl groups diminishes activity, especially using electron-attractor groups as $\mathrm{NO}_{2}$ [41]. A similar behavior is appreciated in the results of flavones $\mathbf{3} \mathbf{b}, \mathbf{3} \mathbf{c}$, and $\mathbf{3} \mathbf{d}$, with $\mathbf{3} \mathbf{b}$ showing good activity with an $\mathrm{EC}_{50}$ of $0.1 \mu \mathrm{g} / \mathrm{mL}$ in DPPH and $0.3 \mu \mathrm{g} / \mathrm{mL}$ in ABTS, in both situations surpassing the positive control; again, it was observed that the presence of electron-attractor groups lowers the activity. Analogs $\mathbf{2 h}$ and $\mathbf{3 h}$ with $p$-Cl substitutions did not show considerable activity when compared to the controls.

The influence of electron-donor groups, like methoxyl, can be analyzed with the $\mathbf{2 e}(p-\mathrm{OMe}), \mathbf{2 f}$ $(m$-OMe), and $2 \mathrm{~g}(p, m$-OMe) analogs; the presence of these groups did not favor the activity, getting concentrations of 100,170 , and $5 \times 10^{4} \mu \mathrm{g} / \mathrm{mL}$, respectively, in DPPH assay, having a similar behavior of the flavones $3 \mathbf{e}-\mathrm{g}$, with $\mathrm{EC}_{50}$ of 30,39 , and $410 \mu \mathrm{g} / \mathrm{mL}$. These results may appear to contrast with the reported by many authors where methoxyl groups are considered an important factor for the antioxidant activity [42,43], however, this is the case where methoxyl groups help with the lipophilicity of the molecule, for example, when the antioxidant activity is measured by the lipid peroxidation activity assay [39]. When employing DPPH test, the transfer of acid protons is required; this implies that just as in the case of the chalcones, we consider indispensable the presence of at least one hydroxyl group, explaining the lack of scavenging activity for the synthesized compounds with only methoxyl groups $(3 \mathbf{e}-\mathbf{g})$.

Molecules $2 \mathbf{i}-3 \mathbf{i}$, both with $p$-dimethylamino substituent showed excellent results with an $\mathrm{EC}_{50}$ of 9.3 and $0.25 \mu \mathrm{g} / \mathrm{mL}$, respectively, in the DPPH assay, having an $\mathrm{EC}_{50}$ of $6.9 \times 10^{-2}$ and $1.9 \times 10^{-3} \mu \mathrm{g} / \mathrm{mL}$ in ABTS; substituents that increase the electronic density, like dimethylamine groups, show potent antioxidant activity that is attributed to the resonance effect involving the pair of electrons of the heteroatom [44].

The $\beta$-carotene decolorization assay brings an outlook to the capacity of an antioxidant to inhibit the lipid peroxidation initiated by the hydrogen abstraction or addition of oxygen radical [45]. The chalcones $\mathbf{2} \mathbf{c}$ and $\mathbf{2} \mathbf{d}$ with hydroxyl substitution showed good activity in this technique with percentages of activity between $76-84 \%$, while the flavones with the same substitution showed results between $67-77 \%$. Undoubtedly, the presence of methoxyl groups in the analogs B ring favored the antioxidant activity, the chalcones $\mathbf{2 e}$ and $\mathbf{2} \mathbf{f}$ exhibit $99 \%$ of antioxidant activity, while the flavones $3 \mathbf{e}$, 3f, and $3 g$ present results of 82,99 and $84 \%$ respectively, all of them better than $\alpha$-tocopherol $(60 \%)$. This allows for determining that the presence of electron-donor groups in the B ring benefits this activity $[46,47]$.

From all the synthesized analogs, it is important to highlight the results obtained for $\mathbf{2} \mathbf{j}, \mathbf{2} \mathbf{k}, \mathbf{3} \mathbf{j}$, and $\mathbf{3 k}$, which have anthracene and furan substitutions, while they presented antiradical activity at very low concentrations in the DPPH assay, with $\mathrm{EC}_{50}$ of 1.7 and $5.4 \times 10^{-3} \mu \mathrm{g} / \mathrm{mL}$ for $\mathbf{2} \mathbf{j}$ and $\mathbf{2 k}$, and $1 \times 10^{-8}$ and $0.1 \mu \mathrm{g} / \mathrm{mL}$ for $3 \mathbf{j}$ and $\mathbf{3 k}$, being far better than the control quercetin $(3 \mu \mathrm{g} / \mathrm{mL})$.

These same four molecules showed a high antioxidant activity in the $\beta$-carotene technique, with results of $98 \%$ and $88 \%$ for the $2 \mathbf{j}$ and $\mathbf{2 k}$ chalcones, while the $\mathbf{3} \mathbf{j}$ and $3 \mathbf{k}$ flavones had $99 \%$ and $85 \%$, respectively, surpassing the $60 \%$ antioxidant activity of $\alpha$-tocopherol. It is known that the oxidation 
of polycyclic aromatic hydrocarbons, such as anthracene generates anthraquinone type products, with 9,10-anthraquinone and 9-hydroxyanthrone being the more abundant [48], the basic skeleton for anthraquinones and anthrone, which provides them the capacity to act as electron acceptors (electrophiles); furthermore, their high conjugation gives them great stability (by resonance means), potentiating the antioxidant activity $[49,50]$. On other hand, the oxidation of furans gives as a result oxyfuranones in the case of monosubstitutions, or diketones in the case of 2,5-disubstituted furans [51]; Y. Sugiyama et al. reported the importance of diketonic systems in the antioxidant activity, both in their keto or enol form [52].

\subsection{Acetylcholinesterase Inhibitory Assay}

The acetylcholinesterase inhibitory activity of the synthesized compounds was evaluated employing Adewusi adaptation of Ellmans spectrophotometrical assay [30], using galantamine as a reference. The results for these experiments are shown with their $\mathrm{IC}_{50}$ values on Table 2. The compounds that were chosen for screening can be divided into two groups based on their structural features: chalcones and flavones.

Table 2. Acetylcholinesterase inhibitory activity of synthesized compounds $\mathbf{2 a} \mathbf{a}-\mathbf{2 k}$ and $\mathbf{3 a} \mathbf{a} \mathbf{- 3 k}$.

\begin{tabular}{cccc}
\hline Compound & IC $_{\mathbf{5 0}}(\boldsymbol{\mu g} / \mathbf{m L})$ & Compound & IC $_{\mathbf{5 0}}(\boldsymbol{\mu g} / \mathbf{m L})$ \\
\hline $\mathbf{2 a}$ & $>150$ & $\mathbf{3 a}$ & $>150$ \\
$\mathbf{2 b}$ & $>150$ & $\mathbf{3 b}$ & $61.2 \pm 1.39$ \\
$\mathbf{2}$ & $52.7 \pm 11.98$ & $\mathbf{3 c}$ & $78.4 \pm 1.92$ \\
$\mathbf{2} \mathbf{d}$ & $21.5 \pm 2.61$ & $\mathbf{3 d}$ & $26.8 \pm 5.91$ \\
$\mathbf{2}$ & $>150$ & $\mathbf{3 e}$ & $43.5 \pm 4.31$ \\
$\mathbf{2} \mathbf{f}$ & $>150$ & $\mathbf{3 f}$ & $60 \pm 9.96$ \\
$\mathbf{2}$ & $>150$ & $\mathbf{3 g}$ & $43.5 \pm 2.52$ \\
$\mathbf{2}$ & $>150$ & $\mathbf{3 h}$ & $>150$ \\
$\mathbf{2}$ & $80.4 \pm 5.97$ & $\mathbf{3 i}$ & $>150$ \\
$\mathbf{2}$ & $>150$ & $\mathbf{3 j}$ & $77.6 \pm 31.89$ \\
$\mathbf{2}$ & $66.4 \pm 6.15$ & $\mathbf{3 k}$ & $>150$ \\
\hline \multicolumn{4}{c}{$0.574 \pm 0.07$} \\
\hline
\end{tabular}

Chalcones are 1,3-diphenyl-2-propene-1-one systems, in which two aromatic rings are linked by a three carbon $\alpha, \beta$-unsaturated carbonyl system. The compounds with this backbone have been reported to possess various biological activities [53]. The $\mathrm{IC}_{50}$ values of these compounds are listed in Table 2. Hasan et al. [54] reported that hydroxyl groups in ortho position of the A ring of chalcones, are an important structural element in the AChE inhibitory activity; however, in our study all the synthesized chalcones had the $o-\mathrm{OH}$ substitution in their A ring, and most of them displayed no inhibition effect in the tested concentration scale. Compounds $\mathbf{2 c}, \mathbf{2} \mathbf{i}$, and $\mathbf{2 k}$ showed an inhibitory effect with $\mathrm{IC}_{50}$ values of $52.7,80.4$, and $66.4 \mu \mathrm{g} / \mathrm{mL}$, respectively, in contrast to the rest of molecules that presented results above $150 \mu \mathrm{g} / \mathrm{mL}$.

On the other hand, results of the flavone group 3a-3k that are based upon a fifteen-carbon skeleton consisting of two benzene rings (A and B) linked via a heterocyclic pyrane ring (C) (Figure 1) indicate that they were more active than the chalcone group. Only the compounds $3 \mathbf{a}, 3 \mathbf{h}, 3 \mathbf{i}$, and $3 \mathbf{k}$ presented poor inhibition as compared to the rest of flavones that, in the majority, have hydroxyl or methoxyl groups in their B ring, where compounds $\mathbf{3 e}, \mathbf{3 g}$, $\mathbf{3 f}$, and $\mathbf{3 b}$ outstand with an $\mathrm{IC}_{50}$ of $43.5,43.5,60.0$, and $61.2 \mu \mathrm{g} / \mathrm{mL}$, respectively; however, compound $3 \mathbf{d}$ with $o-\mathrm{OH}, m-\mathrm{NO}_{2}$ in the $\mathrm{B}$ ring was found to be the best flavone inhibitor of $\mathrm{AChE}$, having an $\mathrm{IC}_{50}$ of $26.8 \mu \mathrm{g} / \mathrm{mL}$.

It has been acknowledged that hydroxyl and methoxyl groups in the A and $\mathrm{B}$ rings of flavones are deeply related to bioactivity and their capacity to protein bonding, being the hydroxyl groups that are favorable for the flavonoids interaction with acetylcholinesterase by hydrogen bonds formation in the enzyme peripheral anionic site (PAS) [55], which would explain the activity showed by compounds $\mathbf{3 b}$, 
$3 \mathbf{c}$, and $3 \mathbf{d}(61.2,78.4$, and $26.8 \mu \mathrm{g} / \mathrm{mL})$. On other hand, the presence of methoxyl groups in flavonoids favors the inhibition effect due to the interaction with residue Trp279, located in the entrance to the active site (in the PAS), this could explain the results obtained for analogs $3 \mathbf{e}-\mathbf{3 g}$.

While we can notice a general improvement of the results with the presence of hydroxyl and methoxyl groups in the chalcones B ring, Sukumaran et al. [56] mentions that, in 2'-hydroxychalcones, the AChE inhibition is generally favored with halogens in the B ring. Nevertheless, their chalcone with chlorine in $p$-position of the B ring did not showed significant activity, molecule that corresponds with our compound $\mathbf{2 h}$, from which we neither detect activity in the tested concentration scale.

As can be noticed, chalcone $\mathbf{2} \mathbf{d}$ and flavone $3 \mathbf{d}$, both with $o-\mathrm{OH}$ and $m-\mathrm{NO}_{2}$ substitutions, were the most active compounds with $\mathrm{IC}_{50}$ values of 21.5 and $26.8 \mu \mathrm{g} / \mathrm{mL}$, respectively, these results suggest that the presence of the nitro group in both molecules enhances the inhibitory activity; it has been previously reported that the introduction of basic or permanently charged nitrogen atoms and aromatic systems, are common structural characteristics in molecules for their interaction with the binding sites of acetylcholinesterase [57].

\subsection{Molecular Docking Analysis}

For the molecular docking studies, the compounds with the best in vitro results $\mathbf{2} \mathbf{d}$ and $\mathbf{3 d}$ were selected in order to gain insight of their interactions with the AChE receptor and explain their biological results. AutoDock Vina was selected to perform the in silico analysis, using the crystallographic structure of the Pacific electric ray Torpedo californica AChE (TcAChE) available from Protein Data Bank (PDB ID:1EVE) as its similarity with human AChE allows for employing it in docking studies [58]. Having selected 1EVE, a docking grid was generated in the active site region defined by the co-crystallized receptor's ligand donepezil. To assure effectiveness of the analysis, it was necessary to validate the method; for this, a re-docking of the co-crystallized donepezil was realized with the same parameters intended to use for our ligands. We compared the best result pose for donepezil with the co-crystallized one obtaining a RMSD of 1.1, which, being lower than 1.5-2 $\AA$, is considered to be a successful analysis, suitable for predicting ligand poses [59].

In Table 3, the resulting scores of the docking for compounds $\mathbf{2} \mathbf{d}$ and $\mathbf{3 d}$ with the inhibition constants can be observed, which AutoDock calculates by the formula:

$$
\mathrm{Ki}=\exp (\Delta \mathrm{G} \times 1000 \mathrm{Rcal} \times \mathrm{TK})
$$

$\Delta G$ is the docking binding energy, $R_{c a l}$ value is 1.98719 , and $T_{K}$ value is 298.15 . In the in vitro results, chalcone $2 \mathbf{d}$ was the most active synthesized compound with an $\mathrm{IC}_{50}$ of $21.5 \mu \mathrm{g} / \mathrm{mL}$, followed by the flavone $3 \mathbf{d}(26.8 \mu \mathrm{g} / \mathrm{mL})$; accordingly, the docking gave a better score for $\mathbf{2} \mathbf{d}(-10.2 \mathrm{kcal} / \mathrm{mol})$ against $3 \mathrm{~d}(-9.78 \mathrm{kcal} / \mathrm{mol})$. The more negative the docking score (binding energy value), the better affinity the ligand has for the receptor.

Table 3. Docking scores for compounds $\mathbf{2 d}$ and $\mathbf{3 d}$.

\begin{tabular}{cccc}
\hline Compound & $\begin{array}{c}\text { Binding Energy } \\
\text { (kcal/mol) }\end{array}$ & $\begin{array}{c}\text { Inhibition Constant } \\
\text { (nM) }\end{array}$ & $\begin{array}{c}\text { Principal Residues } \\
\text { Interactions }\end{array}$ \\
\hline 2d & -10.2 & 33.37 & Trp84, Tyr334, Tyr130 \\
3d & -9.78 & 67.79 & Trp84, Tyr334, Tyr130 \\
\hline
\end{tabular}

Analysis of the poses generated showed us that our compounds interact with AChE mostly by $\pi-\pi$ interactions with the active site residues.

The active site of $\mathrm{AChE}$ consists in a cavity or gorge in the receptor surface that is approximately $20 \AA$ Aeep; besides the catalytic triad of the enzyme (Ser200, His440, and Glu327), which is located at the bottom of the gorge, several residues have been identified as important zones for the interaction of ligands, such as Trp84, Phe330, Glu199, and Gly441 that make the central anionic site (CAS), and 
Trp279, Tyr334, Tyr121, and Tyr70 at the peripheral anionic site (PAS) [60,61]. Both $\mathbf{2 d}$ and $\mathbf{3 d}$ poses are observed nearer to the CAS region, as seen in the Figure 3.

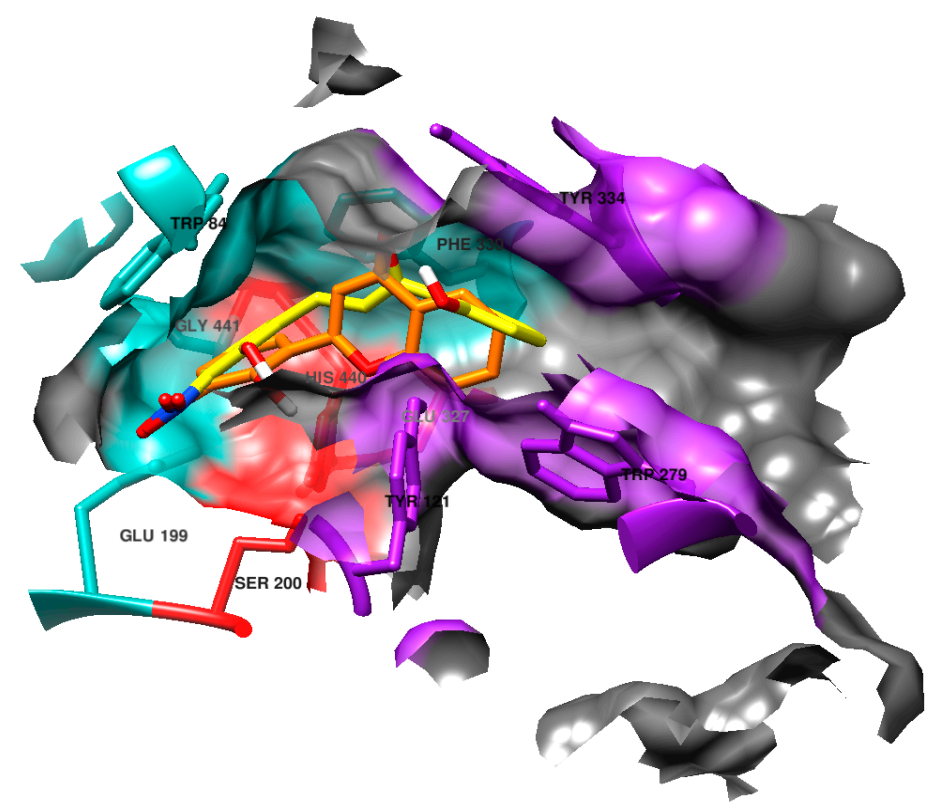

Figure 3. Gorge of 1 EVE depicting compounds $\mathbf{2} \mathbf{d}$ (yellow) and $\mathbf{3 d}$ (orange) position and the catalytic triad (red), central anionic site (CAS) (blue), and peripheral anionic site (PAS) (purple).

The best pose generated for $\mathbf{2} \mathbf{d}$ does not form hydrogen bonds to the nearest residues; although, one oxygen of the nitro group is near the hydrogen of Tyr130, at only $2.7 \AA$, the angle between them does not allow a proper hydrogen bond. A small turn of the nitro or hydroxyl groups would be needed for this to happen, but a weak interaction between them might be helping the interaction of $\mathbf{2} \mathbf{d}$ with this residue. The clearer interactions for $\mathbf{2} \mathbf{d}$ appear with Trp84, a residue that has been reported as an important one for ligand interactions (especially with quaternary groups from acetylcholine and other compounds) [58,62]. In this case, Trp84 is near the positively charged nitrogen of the nitro group, although its position appears to be almost over the B ring of the chalcone, allowing for $\pi-\pi$ stacking at 3.7 ̊.. Another $\pi-\pi$ stacking can be observed between the A ring of $\mathbf{2} \mathbf{d}$ and Tyr334 in the PAS region, with $4 \AA$ among the aromatic rings (Figure 4). The side of chalcone $2 \mathrm{~d}$ with the A ring rests in the mostly hydrophobic zone created by Trp334, Phe331, and Phe330, while the side of the B ring points to a less hydrophobic area.

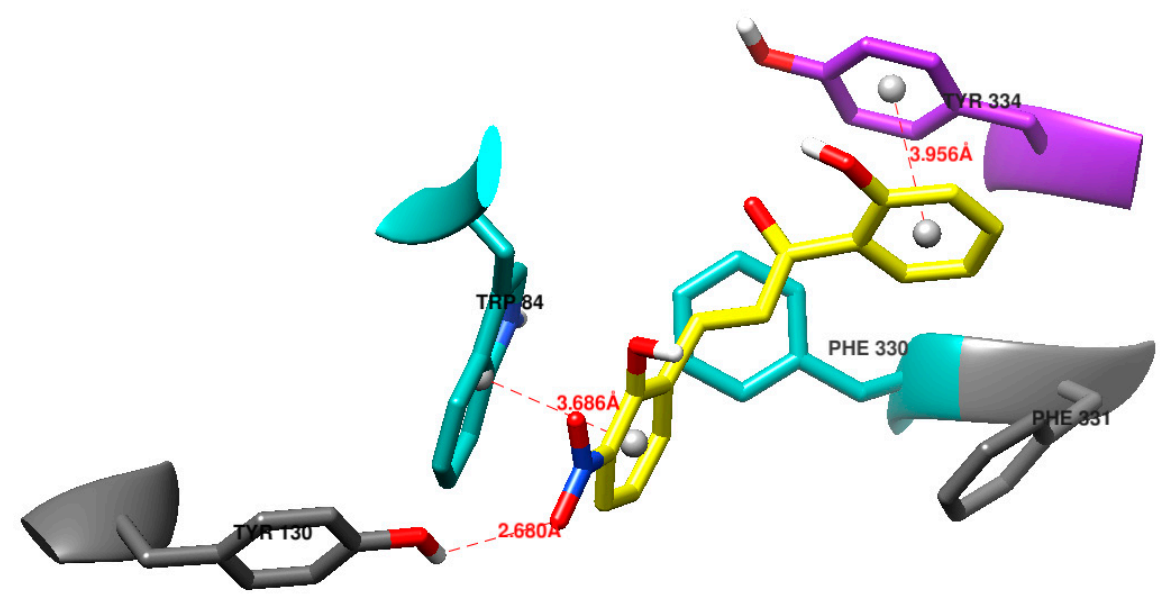

Figure 4. Chalcone $2 \mathrm{~d}$ and its interactions with nearby residues. 
The case for the best generated pose of flavone $\mathbf{3 d}$ is very similar to its chalcone counterpart. Although a formal hydrogen bond is not detected by the Chimera software due to the angle between them, one oxygen of the nitro group is near the hydrogen of Tyr130, at $2.6 \AA$, so a weak interaction among these atoms could be possible (Figure 5). The nitro group is near to Trp84, but the $B$ ring appears to have a better interaction with this residue in form of $\pi-\pi$ stacking at a distance of $4 \AA$. The $\mathrm{A}$ ring has proximity to Tyr334, even though with the new ring formation the flavone $\mathbf{3 d}$ has a more rigid position for the A ring, which now appears to have with its edge a perpendicular $\pi-\pi$ interaction with Tyr334 at 3.6 $\AA$. The $C$ ring is oriented to Phe 330 with a distance among them of $4.4 \AA$. As the chalcone $2 \mathbf{d}$ did, the $B$ ring with the nitro group of the flavone $3 \mathbf{d}$ is oriented to the bottom of the gorge, in a less hydrophobic area, while the rest of the structure is towards the hydrophobic zone of Trp334, Phe331, and Phe330, as can be seen in Figure 6.

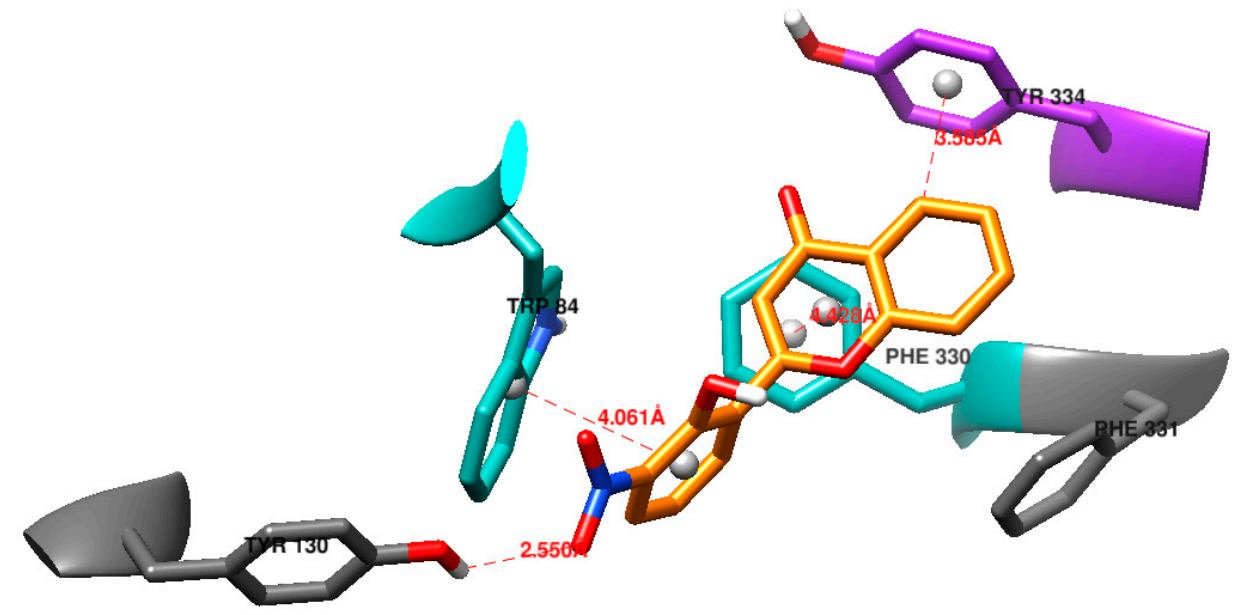

Figure 5. Flavone 3d and its interactions with nearby residues.

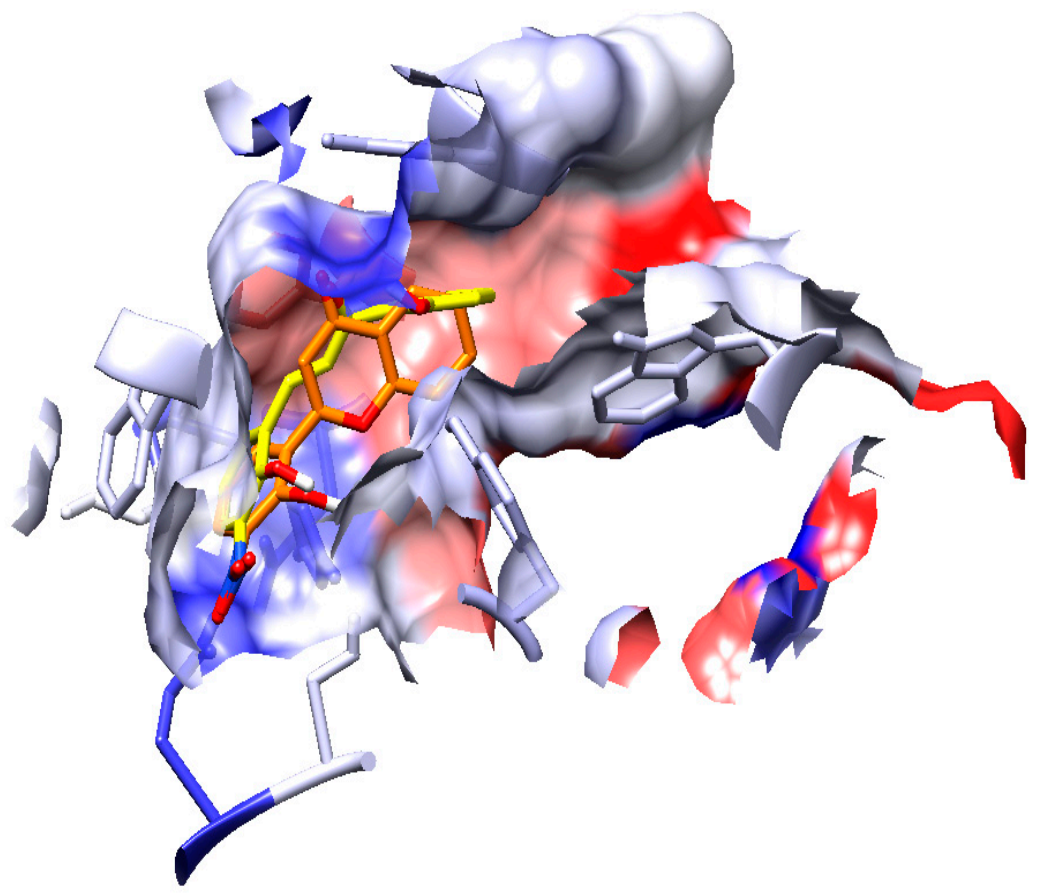

Figure 6. Compounds $2 \mathrm{~d}$ (yellow) and $3 \mathrm{~d}$ (orange) at the gorge of TcAChE. The color of the surface of the receptor indicates hydrophilicity (blue) passing by white to red (hydrophobicity). 


\section{Conclusions}

In conclusion, for the three analyzed techniques, the flavone type analogs showed a better antioxidant profile when compared to the chalcones, however this does not mean that the latter were inactive since many of them resulted highly active. Surprisingly, the compounds $\mathbf{2} \mathbf{j}, \mathbf{2} \mathbf{k}, \mathbf{3} \mathbf{j}$, and $\mathbf{3 k}$ presented a high potential as antioxidant agents, this could be due to the quinone and oxyfuranone type products that are generated after their oxidation. As previous reports have stated, the presence of hydroxyl groups favors the antiradical activity mediated by hydrogen donation, as it could be noticed that compounds $\mathbf{2} \mathbf{b}$ and $\mathbf{3} \mathbf{b}$ surpassed the positive controls in both DPPH and ABTS assays; in the $\beta$-carotene technique, the electron-donor substitutions were the most active.

On the other hand, in the AChE inhibition assay, the better results from the chalcone and flavone families (compounds $\mathbf{2} \mathbf{d}$ and $\mathbf{3 d}$ ) share the nitro functional group, with $\mathrm{IC}_{50}$ values of 21.5 and $26.8 \mu \mathrm{g} / \mathrm{mL}$, respectively. The docking results suggest that the principal interactions compounds $\mathbf{2 d}$ and $3 \mathbf{d}$ have with AChE active site are mostly $\pi-\pi$ stackings. This can be observed with residues Trp84 and Tyr334 from the CAS and PAS, which are known binding zones of the enzyme.

Although further in vivo testing must be performed, our results represent an important step towards the identification of improved antioxidants and acetylcholinesterase inhibitors.

Supplementary Materials: The following are available online at http://www.mdpi.com/2076-3417/9/3/410/s1, Figure S1: ${ }^{1} \mathrm{H}-\mathrm{NMR}$ Compound 2e, Figure S2: ${ }^{13} \mathrm{C}-\mathrm{NMR}$ Compound 2e, Figure S3: COSY-Compound 2e, Figure S4: HSQC-Compound 2e, Figure S5: HMBC-Compound 2e, Figure S6: ${ }^{1} \mathrm{H}-\mathrm{NMR}$ Compound 3e, Figure S7: ${ }^{13} \mathrm{C}-\mathrm{NMR}$ Compound 3e, Figure S8: COSY Compound 3e, Figure S9: HSQC Compound 3e, Figure S10: HMBC Compound 3e, Figure S11: ${ }^{1} \mathrm{H}-\mathrm{NMR}$ Compound 2k, Figure S12: ${ }^{13} \mathrm{C}-\mathrm{NMR}$ Compound 2k, Figure S13: ${ }^{1} \mathrm{H}-\mathrm{NMR}$ Compound 3k, Figure S14: ${ }^{13} \mathrm{C}-\mathrm{NMR}$ Compound 3k, Figure S15: ${ }^{1} \mathrm{H}-\mathrm{NMR}$ Compound 2h, Figure S16: ${ }^{13} \mathrm{C}-\mathrm{NMR}$ Compound 2h, Figure S17: ${ }^{1} \mathrm{H}-\mathrm{NMR}$ Compound 3h, Figure S18: ${ }^{13} \mathrm{C}-\mathrm{NMR}$ Compound 3h, Figure S19: ${ }^{1} \mathrm{H}-\mathrm{NMR}$ Compound 2d, Figure S20: ${ }^{13} \mathrm{C}-\mathrm{NMR}$ Compound 2d, Figure S21: ${ }^{1} \mathrm{H}-\mathrm{NMR}$ Compound 2j, Figure S22: ${ }^{13} \mathrm{C}-\mathrm{NMR}$ Compound $\mathbf{2 j}$, Figure S23: ${ }^{1} \mathrm{H}-\mathrm{NMR}$ Compound $\mathbf{3} \mathbf{j}$, Figure S24: ${ }^{13} \mathrm{C}-\mathrm{NMR}$ Compound $\mathbf{3} \mathbf{j}$.

Author Contributions: Conceptualization, I.C.-G. supervised the whole study; L.D.-R. and A.E.-C. carried out the chemical synthesis; L.D.-R. and R.H.-M. performed the chemical assay, I.A.R.-E. and D.C.-V. performed the spectroscopic data; M.A.R. collaborated in the discussion and interpretation of the results; L.D.-R., A.E.-C. and V.G.-G. wrote the manuscript; R.S.-A. and N.W.d.T. performed the enzymatic assay; A.E.-C and M.A.R. carried out the in silico study. All authors read and approved the final manuscript.

Acknowledgments: We gratefully acknowledge the Facultad de Ciencias Químicas e Ingeniería, Universidad Autónoma de Baja California for the financing given for the realization of this project. Additionally, to the Consejo Nacional de Ciencia y Tecnología (CONACYT) for ITT NMR facilities (Grant INFR-2011-3-173395).

Conflicts of Interest: The authors declare no conflict of interest.

\section{References}

1. Ray, P.D.; Huang, B.W.; Tsuji, Y. Reactive oxygen species (ROS) homeostasis and redox regulation in cellular signaling. Cell. Signal. 2012, 24, 981-990. [CrossRef] [PubMed]

2. Greeff, J.; Joubert, J.; Malan, S.F.; Van Dyk, S. Antioxidant properties of 4-quinolones and structurally related flavones. Bioorg. Med. Chem. 2012, 20, 809-818. [CrossRef] [PubMed]

3. Niu, Y.; Cao, W.; Zhao, Y.; Zhai, H.; Zhao, Y.; Tang, X.; Chen, Q. The levels of oxidative stress and antioxidant capacity in hibernating Nanorana parkeri. Comp. Biochem. Physiol. A Mol. Integr. Physiol. 2018, 219-220, 19-27. [CrossRef] [PubMed]

4. Giustarini, D.; Dalle-Donne, I.; Tsikas, D.; Rossi, R. Oxidative stress and human diseases: Origin, link, measurement, mechanisms, and biomarkers. Crit. Rev. Clin. Lab. Sci. 2009, 46, 241-281. [CrossRef] [PubMed]

5. Halliwell, B. Oxidative stress and neurodegeneration: Where are we now? J. Neurochem. 2006, 97, 1634-1658. [CrossRef] [PubMed]

6. Pham-Huy, L.A.; He, H.; Pham-Huy, C. Free Radicals, Antioxidants in Disease and Health. Int. J. Biomed. Sci. 2008, 4, 89-96. [PubMed]

7. Abdoh, T.; Khalil, A.A.; Awais, U.I.; Jia, Q.; Na, L.; Kamal, H.; Nirmala, K.; Lei, H.; Ding, Q. Antioxidant effects and mechanism of silymarin in oxidative stress induced cardiovascular diseases. Biomed. Pharm. 2018, 102, 689-698. [CrossRef] 
8. Angoa Pérez, M.; Rivas Arancibia, S. Estrés oxidativo y neurodegeneración: ¿causa o consecuencia? Arch. Neurocien. 2007, 12, 45-54.

9. Tran, T.D.; Nguyen, T.C.V.; Nguyen, N.S.; Nguyen, D.M.; Nguyen, T.T.H.; Le, M.T.; Thai, K.M. Synthesis of novel chalcones as acetylcholinesterase inhibitors. Appl. Sci. 2016, 6, 198. [CrossRef]

10. Díaz-Hung, M.L.; González Fraguela, M.E. El estrés oxidativo en las enfermedades neurológicas: ¿causa o consecuencia? Oxidative stress in neurological diseases: Cause or effect? Neurologia 2014, 29, 451-452. [CrossRef]

11. Martínez-Lazcano, J.C.; Boll-Woehrlen, M.C.; Hernández-Melesio, M.; Rubio-Osornio, M.; Sánchez-Mendoza, M.; Ríos, C. Radicales libres y estrés oxidativo en las enfermedades neurodegenerativas. Mensaje Bioquim 2010, 34, 43-59.

12. Uriarte-Pueyo, I.; Calvo, M.I. Flavonoids as acetylcholinesterase inhibitors. Curr. Med. Chem. 2011, 18, 5289-5302. [CrossRef] [PubMed]

13. Tan, N.D.; Dao, T.T. Synthesis, Antioxidant and Antimicrobial Activities of a Novel Series of Chalcones, Pyrazolic Chalcones, and Allylic Chalcones. Pharmacol. Pharm. 2011, 2, 282-288. [CrossRef]

14. Serafini, M.; Peluso, I.; Raguzzini, A. Flavonoids as anti-inflammatory agents. Proc. Nutr. Soc. 2010, 69, 273-278. [CrossRef] [PubMed]

15. Davila, J.C.; Lenherr, A.; Acosta, D. Protective effect of flavonoids on drug-induced hepatotoxicity in vitro. Toxicology 1989, 57, 267-286. [CrossRef]

16. Parmar, N.S.; Parmar, S. Anti-ulcer potential of flavonoids. Indian J. Physiol. Pharm. 1998, 42, 343-351.

17. Ribeiro, D.; Freitas, M.; Tomé, S.M.; Silva, A.M.; Laufer, S.; Lima, J.L.; Fernandes, E. Flavonoids inhibit COX-1 and COX-2 enzymes and cytokine/chemokine production in human whole blood. Inflammation 2015, 38, 858-870. [CrossRef]

18. Lin, S.; Zhang, G.; Liao, Y.; Pan, J.; Gong, D. Dietary Flavonoids as Xanthine Oxidase Inhibitors: Structure-Affinity and Structure-Activity Relationships. J. Agric. Food. Chem. 2015, 63, 7784-7794. [CrossRef]

19. Schewe, T.; Kühn, H.; Sies, H. Flavonoids of cocoa inhibit recombinant human 5-lipoxygenase. J. Nutr. 2002, 132, 1825-1829. [CrossRef]

20. Ko, W.C.; Shih, C.M.; Lai, Y.H.; Chen, J.H.; Huang, H.L. Inhibitory effects of flavonoids on phosphodiesterase isozymes from guinea pig and their structure-activity relationships. Biochem. Pharm. 2004, 68, 2087-2094. [CrossRef]

21. Lani, R.; Hassandarvish, P.; Shu, M.H.; Phoon, W.H.; Chu, J.J.; Higgs, S.; Vanlandingham, D.; Abu, B.S.; Zandi, K. Antiviral activity of selected flavonoids against Chikungunya virus. Antivir. Res. 2016, 133, 50-61. [CrossRef] [PubMed]

22. Peterson, J.J.; Dwyer, J.T.; Jacques, P.F.; McCullough, M.L. Associations between flavonoids and cardiovascular disease incidence or mortality in European and US populations. Nutr. Rev. 2012, 70, 491-508. [CrossRef] [PubMed]

23. Chang, H.; Mi, M.; Ling, W.; Zhu, J.; Zhang, Q.; Wei, N.; Zhou, Y.; Tang, Y.; Yuan, J. Structurally related cytotoxic effects of flavonoids on human cancer cells in vitro. Arch. Pharm. Res. 2008, 31, 1137-1144. [CrossRef] [PubMed]

24. Williams, R.J.; Spencer, J.P.; Rice-Evans, C. Flavonoids: Antioxidants or signalling molecules? Free Radic. Biol. Med. 2004, 36, 838-849. [CrossRef] [PubMed]

25. Seyoum, A.; Asres, K.; El-Fiky, F.K. Structure-radical scavenging activity relationships of flavonoids. Phytochemistry 2006, 67, 2058-2070. [CrossRef] [PubMed]

26. Salazar-Aranda, R.; Granados-Guzmán, G.; Pérez-Meseguer, J.; González, G.M.; Waksman de Torres, N. Activity of Polyphenolic Compounds against Candida glabrata. Molecules 2015, 20, 17903-17912. [CrossRef]

27. Re, R.; Pellegrini, N.; Proteggente, A.; Pannala, A.; Yang, M.; Rice-Evans, C. Antioxidant activity applying an improved ABTS radical cation decolorization assay. Free Radic. Biol. Med. 1999, 26, 1231-1237. [CrossRef]

28. Kuskoski, E.M.; Asuero, A.G.; Troncoso, A.M.; Mancini-Filho, J.; Fett, R. Aplicación de diversos métodos químicos para determinar actividad antioxidante en pulpa de frutos. Food Sci. Technol. 2005, 25, 726-732. [CrossRef]

29. Burda, S.; Oleszek, W. Antioxidant and Antiradical Activities of Flavonoids. J. Agric. Food Chem. 2001, 49, 2774-2779. [CrossRef]

30. Adewusi, E.A.; Moodley, N.; Steenkamp, V. Antioxidant and acetylcholinesterase inhibitory activity of selected southern African medicinal plants. S. Afr. J. Bot. 2011, 77, 638-644. [CrossRef] 
31. Pettersen, E.F.; Goddard, T.D.; Huang, C.C.; Couch, G.S.; Greenblatt, D.M.; Meng, E.C.; Ferrin, T.E. UCSF Chimera-A visualization system for exploratory research and analysis. J. Comput. Chem. 2004, 25, 1605-1612. [CrossRef]

32. Wang, J.; Wang, W.; Kollman, P.A.; Case, D.A. Automatic atom type and bond type perception in molecular mechanical calculations. J. Mol. Graph. Model. 2006, 25, 247-260. [CrossRef]

33. Sanner, M.F. Python: A programming language for software integration and development. J. Mol. Graph. Model. 1999, 17, 57-61. [CrossRef]

34. Trott, A.; Olson, A.J. AutoDock Vina: Improving the speed and accuracy of docking with a new scoring function, efficient optimization, and multithreading. J. Comput. Chem. 2010, 31, 455-461. [CrossRef]

35. Zhuang, C.; Zhang, W.; Sheng, C.; Zhang, W.; Xing, C.; Miao, Z. Chalcone: A Privileged Structure in Medicinal Chemistry. Chem. Rev. 2017, 117, 7762-7810. [CrossRef]

36. Lee, Y.H.; Jeon, S.-H.; Kim, S.H.; Kim, C.; Lee, S.-J.; Koh, D.; Lim, Y.; Ha, K. A new synthetic chalcone derivative, 2-hydroxy-3',5,5'-trimethoxychalcone (DK-139), suppresses the Toll-like receptor 4-mediated inflammatory response through inhibition of the Akt/NF-kB pathway in BV2 microglial cells. Exp. Mol. Med. 2012, 44, 369-377. [CrossRef]

37. Lokhande, P.D.; Sakate, S.S.; Taksande, K.N.; Navghare, B. Dimethylsulfoxide-iodine catalysed deprotection of 2'-allyloxychalcones: Synthesis of flavones. Tetrahedron Lett. 2005, 46, 1573-1574. [CrossRef]

38. Shenvi, S.; Kumar, K.; Hatti, S.K.; Rijesh, K.; Diwakar, L.; Reddy, G.C. Synthesis, anticancer and antioxidant activities of 2,4,5-trimethoxy chalcones and analogues from asaronaldehyde: Structure-activity relationship. Eur. J. Med. Chem. 2013, 62, 435-442. [CrossRef]

39. Joshi, A.J.; Gaghwal, M.K.; Joshi, U.J.; D’Mello, P.; Sinha, R.; Govil, G. Synthesis of B-ring substituted flavones and evaluation of their antitumor and antioxidant activities. Med. Chem. Res. 2013, 22, 4293-4299. [CrossRef]

40. Rezk, B.M.; Haenen, G.R.; Van der Vijgh, W.J.; Bast, A. The antioxidant activity of phloretin: The disclosure of a new antioxidant pharmacophore in flavonoids. Biochem. Biophys. Res. Commun. 2002, 295, 9-13. [CrossRef]

41. Pan, Y.; Chen, Y.; Li, Q.; Yu, X.; Wang, J.; Zheng, J. The synthesis and evaluation of novel hydroxyl substituted chalcone analogs with in vitro anti-free radicals pharmacological activity and in vivo anti-oxidation activity in a free radical-injury Alzheimer's model. Molecules 2013, 18, 1693-1703. [CrossRef]

42. Jung, J.-C.; Lee, Y.; Min, D.; Jung, M.; Oh, S. Practical synthesis of chalcone derivatives and their biological activities. Molecules 2017, 22, 1872. [CrossRef]

43. Sulpizio, C.; Roller, A.; Giester, G.; Rompel, A. Synthesis, structure, and antioxidant activity of methoxy- and hydroxyl-substituted 2'-aminochalcones. Mon. Chem. 2016, 147, 1747-1758. [CrossRef]

44. Scotti, L.; Mendonça, J.J.B.; Magalhaes, M.D.R.; Sobral da Silva, M.; Pitta, I.R.; Scotti, M.T. SAR, QSAR and Docking of Anticancer Flavonoids and Variants: A Review. Curr. Top. Med. Chem. 2012, 12, 2785-2809. [CrossRef]

45. Nickavar, B.; Esbati, N. Evaluation of the antioxidant capacity and phenolic content of three Thymus species. J. Acupunct. Meridian Stud. 2012, 5, 119-125. [CrossRef]

46. Detsi, A.; Majdalani, M.; Kontogiorgis, C.A.; Hadjipavlou-Litina, D.; Kefalas, P. Natural and synthetic 2'-hydroxy-chalcones and aurones: Synthesis, characterization and evaluation of the antioxidant and soybean lipoxygenase inhibitory activity. Bioorg. Med. Chem. 2009, 17, 8073-8085. [CrossRef]

47. Heijnen, C.G.M.; Haenen, G.R.M.M.; Oostveen, R.M.; Stalpers, E.M.; Bast, A. Protection of Flavonoids Against Lipid Peroxidation: The Structure Activity Relationship Revisited. Free Radic. Res. 2002, 36, 575-581. [CrossRef]

48. Cordeiro, D.S.; Corio, P. Electrochemical and photocatalytic reactions of polycyclic aromatic hydrocarbons investigated by Raman spectroscopy. J. Braz. Chem. Soc. 2009, 20, 80-87. [CrossRef]

49. Fouillaud, M.; Caro, Y.; Venkatachalam, M.; Grondin, I.; Dufossé, L. Anthraquinones. In Phenolic Compounds in Food: Characterization and Analysis, 1st ed.; Nollet, L.M.L., Gutiérrez-Uribe, J.A., Eds.; CRC Press: Boca Raton, FL, USA, 2018; Volume 1, pp. 131-172.

50. Cock, I.E. The Genus Aloe: Phytochemistry and therapeutic uses including treatments for gastrointestinal conditions and chronic inflammation. In Novel Natural Products: Therapeutic Effects in Pain, Arthritis and Gastro-Intestinal Diseases, 1st ed.; Rainsford, K.D., Powanda, M.C., Whitehouse, M.W., Eds.; Springer: Basel, Switzerland, 2015; Volume 70, pp. 179-235.

51. Höfer, M.; Moszner, N.; Liska, R. Oxygen scavengers and sensitizers for reduced oxygen inhibition in radical photopolymerization. J. Polym. Sci. A Polym. Chem. 2008, 46, 6916-6927. [CrossRef] 
52. Sugiyama, Y.; Kawakishi, S.; Osawa, T. Involvement of the beta-diketone moiety in the antioxidative mechanism of tetrahydrocurcumin. Biochem. Pharmacol. 1996, 52, 519-525. [CrossRef]

53. Chavan, B.B.; Gadekar, A.S.; Mehta, P.P.; Vawhal, P.K.; Kolsure, A.K.; Chabukswar, A.R. Synthesis and medicinal significance of chalcones-A review. Asian J. Biomed. Pharm. Sci. 2016, 6, 1-7.

54. Hasan, A.; Khan, K.M.; Sher, M.; Maharvi, G.M.; Nawaz, S.A.; Choudhary, M.I.; Atta-Ur-Rahman; Supuran, C.T. Synthesis and inhibitory potential towards acetylcholinesterase, butyrylcholinesterase and lipoxygenase of some variably substituted chalcones. J. Enzym. Inhib. Med. Chem. 2005, 20, 41-47. [CrossRef]

55. Xie, Y.; Yang, W.; Chen, X.; Xiao, J. Inhibition of flavonoids on acetylcholine esterase: Binding and structure-activity relationship. Food Funct. 2014, 5, 2582-2589. [CrossRef]

56. Sukumaran, S.D.; Chee, C.F.; Viswanathan, G.; Buckle, M.J.C.; Othman, R.; Rahman, N.A.; Chung, L.Y. Synthesis, biological evaluation and molecular modelling of 2 '-hydroxychalcones as acetylcholinesterase inhibitors. Molecules 2016, 21, 955. [CrossRef]

57. Andersson, C.D.; Forsgren, N.; Akfur, C.; Allgardsson, A.; Berg, L.; Engdahl, C.; Qian, W.; Ekström, F.; Linusson, A. Divergent structure-activity relationships of structurally similar acetylcholinesterase inhibitors. J. Med. Chem. 2013, 56, 7615-7624. [CrossRef]

58. Kryger, G.; Silman, I.; Sussman, J.L. Structure of acetylcholinesterase complexed with E2020 (Aricept ${ }^{\circledR)}$ ): Implications for the design of new anti-Alzheimer drugs. Structure 1999, 7, 297-307. [CrossRef]

59. Hevener, K.E.; Zhao, W.; Ball, D.M.; Babaoglu, K.; Qi, J.; White, S.W.; Lee, R.E. Validation of molecular docking programs for virtual screening against dihydropteroate synthase. J. Chem. Inf. Model. 2009, 49, 444-460. [CrossRef]

60. Abdelhameed, R.; Elgawish, M.S.; Mira, A.; Ibrahim, A.K.; Ahmed, S.A.; Shimizu, K.; Yamada, K. Anti-choline esterase activity of ceramides from the Red Sea marine sponge Mycale euplectellioides. RSC Adv. 2016, 6, 20422-20430. [CrossRef]

61. Khoobi, M.; Alipour, M.; Sakhteman, A.; Nadri, H.; Moradi, A.; Ghandi, M.; Emami, S.; Foroumadi, A.; Shafiee, A. Design, synthesis, biological evaluation and docking study of 5-oxo-4,5-dihydropyrano[3,2-c]chromene derivatives as acetylcholinesterase and butyrylcholinesterase inhibitors. Eur. J. Med. Chem. 2013, 68, 260-269. [CrossRef]

62. Neto, D.C.; de Souza Ferreira, M.; da Conceição Petronilho, E.; Lima, J.A.; de Azeredo, S.O.; Brum, J.D.; do Nascimento, C.J.; Villar, J.D. A new guanylhydrazone derivative as a potential acetylcholinesterase inhibitor for Alzheimer's disease: Synthesis, molecular docking, biological evaluation and kinetic studies by nuclear magnetic resonance. RSC Adv. 2017, 7, 33944-33952. [CrossRef] 